\title{
ON HIGHER RANK INSTANTONS \& THE MONOPOLE COBORDISM PROGRAM
}

\author{
RAPHAEL ZENTNER
}

\begin{abstract}
Witten's conjecture suggests that the polynomial invariants of Donaldson are expressible in terms of the Seiberg-Witten invariants if the underlying four-manifold is of simple type. A higher rank version of the Donaldson invariants was introduced by Kronheimer. Before even having been defined, the physicists Mariño and Moore had already suggested that there should be a generalisation of Witten's conjecture to this type of invariants. We study a generalisation of the classical cobordism program to the higher rank situation and obtain vanishing results which gives evidence that the generalisation of Witten's conjecture should hold.
\end{abstract}

\section{INTRODUCTION}

Recently Peter Kronheimer introduced polynomial invariants associated to moduli spaces of anti-selfdual $P U(N)$ connections in Hermitian rank-N-bundles [13. These are generalisations of the Donaldson invariants, but the technical problems are much harder than in the classical situation. Before these invariants were even properly defined, the physicists Mariño and Moore [16 had conjectured that there should be a generalisation of Witten's conjecture for these invariants, implying in particular that they do not contain new differential-topological information. Their argument relies again on physics. Kronheimer computed explicitely his higher rank invariants for manifolds obtained through knot surgery on the K3-surface, confirming the conjecture for this class of examples.

The main important gauge-theoretical invariants of a smooth closed four-manifold are the polynomial invariants of Donaldson [4, derived from anti-selfdual $P U(2)$ connections in rank-2-bundles, and the Seiberg-Witten invariants, derived from the Seiberg-Witten equations 29] which are associated to $\operatorname{Spin}^{c}$ structures on the four-manifold. Kronheimer and Mrowka have proved a structure theorem for the Donaldson invariants [15, showing that for 4-manifolds of 'simple type' the polynomial invariants are specified by certain algebraic-topological data, in particular the intersection form, and a finite set of distinguished cohomology classes in the group $H^{2}(X, \mathbb{Z})$ each coming with some rational coefficient. With this at hand, Witten claims that the polynomial invariants are determined by the Seiberg-Witteninvariants, with the basic classes being the first Chern classes of the Spin ${ }^{c}$-structures with non-trivial Seiberg-Witten-invariant, and with an explicit formula for the rational coefficients [29].

Witten derived this conjecture from correlation functions in quantum field theory and certain limiting behaviours with respect to a certain coupling constant. Mathematicians then tried to derive a proof of the conjecture by a certain cobordism obtained from $P U(2)$ monopoles. Heuristically the idea is as follows. There is a 
circle action on the moduli space of $P U(2)$ monopoles which comes from multiplying the spinor component by a complex number of norm one. The fixed point set of this action consists of the moduli space of $P U(2)$ instantons, and further a finite number of moduli spaces of classical $U(1)$ Seiberg-Witten monopoles. The circle acts freely on the complement of this fixed point locus, and the quotient yields a cobordism between a projective bundle over the moduli space of $P U(2)$ instantons, and projective bundles over the moduli spaces of $U(1)$ Seiberg-Witten monopoles.

Furthermore, the canoncial cohomology class that yields the polynomial invariant of Donaldson extends to the cobordism. The evaluation of this extension on one side, yielding the Donaldson invariant, is therefore equal to the evaluation on the other sides, which should be expressions containing the Seiberg-Witten invariants of the moduli spaces in the fixed point locus. This program was started independently by Pidstrigach and Tyurin [24, and Okonek and Teleman [21, [26]. It was carried on over years by Feehan and Leness 6 , 7, 8, 9, It seems that they have now proved the full conjecture [10].

Our intention is to consider the generalisation of the cobordism program with the perspective of applying it to the generalisation of Witten's conjecture by Mariño and Moore. We introduce $P U(N)$ monopoles: For a given $S_{p i n}{ }^{c}$ structure $\mathfrak{s}$ and a Hermitian rank-N-bundle $E$ on $X$, the configuration space will consist of sections $\Psi$ of the 'twisted spinor bundle' $W^{+}=S_{\mathfrak{s}}^{+} \otimes E$ and by unitary connections $A$ in $E$ with fixed induced connection $\theta$ in the determinant line bundle of $E$. The straightforward generalisation of the $P U(2)$ monopole equations then read:

$$
\begin{aligned}
D_{A}^{+} \Psi & =0 \\
\gamma\left(\left(F_{A}^{+}\right)_{0}\right)-\mu_{0,0}(\Psi) & =0 .
\end{aligned}
$$

Here $\not_{A}^{+}$is the associated Dirac-operator to $A$, the map $\gamma$ is derived from Cliffordmultiplication, $\left(F_{A}^{+}\right)_{0}$ is the self-dual part of the curvature of the $P U(N)$ connection $A$ induced by $A$, and $\mu_{0,0}$ is a quadratic map in the spinor which is explicitely described below. The gauge group of the problem is that of special unitary automorphisms of $E$.

Again, there is a circle action on the moduli space of these $P U(N)$ monopoles which is given by the formula $(z,[\Psi, A]) \mapsto\left[z^{1 / N} \Psi, A\right]$. The moduli space of $P U(N)$ instantons is contained as the locus of monopoles with vanishing spinor, and the other fixed point loci are labelled by a finite number of isomorphism classes of proper subbundles $[F]$ of $E$. An equivalence class $[\Psi, A]$ belongs to the $[F]$-locus $M^{[F]}$ if for each $F \in[F]$ there is a representative $(\Psi, A)$ with the spinor $\Psi$ being a section of $S_{\mathfrak{s}}^{+} \otimes F$ and with the connection $A$ keeps the proper subbundle $F$ invariant. It turns out that if $X$ is simply connected the description of $M^{[F]}$ is particularly simple after fixing one such $F$ : The content of Theorem 2.20 is that we have a 'parametrisation'

$$
M_{\mathfrak{s}, F} \times M_{F^{\perp}}^{a s d} \rightarrow M^{[F]} .
$$

Here $M_{\mathfrak{s}, F}$ is a moduli space of $U(n)$ monopoles with $n=\operatorname{rk}(F)$ having possible values $1 \leq n<N$, and $M_{F^{\perp}}^{a s d}$ is the moduli space of anti-selfdual $P U(N-n)$ connections in $F^{\perp}$. In the case $n=1$ the moduli space $M_{\mathfrak{s}, F}^{U}$ is a classical $U(1)$ Seiberg-Witten moduli space. The map (1) is surjective and is bijective if restricted to the subspaces of the corresponding moduli spaces which consist of elements with 
zero-dimensional stabiliser. In the classical case $N=2$ we can only have $n=1$ and there are no non-trivial $P U(1)$ connections.

Now the components $M^{[F]}$ are the possible contributions to the formula expressing the $P U(N)$ instanton invariant according to the cobordism program indicated above. The generalisation of Witten's conjecture to the $P U(N)$ instanton invariants would follow if only those components $M^{[F]}$ contribute in a non-trivial way for which we have $n=\operatorname{rk}(F)=1$. We give two results that indicate that this should be true - one general but under slightly speculative simplifying assumption, the other on Kähler surfaces.

The first section sets up our configuration space, introduces the above mentioned quadratic map $\mu_{0,0}$ and variations $\mu_{0, \tau}$ of it with a parameter $\tau \in[0,1]$. We derive some important properness property of it. The $P U(N)$ monopole equations are then introduced and it is indicated how to obtain an Uhlenbeck-type compactification of the moduli space. With only minor modifications, we then introduce the $U(n)$ monopole equations. The second section studies the circle action on the moduli space of $P U(N)$ monopoles, analyses its fixed point set and relates it to $U(n)$ monopoles and $P U(N-n)$ instantons. The third section gives our first argument why we expect no contributions from fixed point loci $M_{\mathfrak{s}, F} \times M_{F^{\perp}}^{\text {asd }}$ for $\operatorname{rk}(F)>1$. We prove that under certain assumptions the moduli space $M_{\mathfrak{s}, F}$ is cobordant to the empty space. For the second argument, we analyse the $U(n)$ monopole equations on Kähler surfaces in the forth section. By studying decoupling phenomena, we show that the moduli space becomes empty as soon as we perturb by a non-zero holomorphic two-form.

\section{ACKNOWLEDGEMENTS}

This manuscript constitutes a part of the author's $\mathrm{PhD}$ thesis. He is grateful to his former advisor Andrei Teleman for the care taken in guiding him and the many mathematical discussions involved. He is also indebted to Peter Kronheimer and Kim Frøyshov for discussions on some of the aspects.

\section{Preliminaries, $P U(N)$ and $U(n)$ monopoles}

Here we shall introduce the $P U(N)$ monopole as well as the $U(n)$ monopole equations associated to the data of a Spin $^{c}$-structure $\mathfrak{s}$ and a Hermitian bundle $E \rightarrow X$ on a Riemannian four-manifold $X$. We shall define the monopole equations, the moduli space, and prove a uniform bound on the spinor component of a solution to the monopole equations. We assume knowledge with standard (abelian) SeibergWitten theory as for instance found in the monographs [19, 17] or the lecture notes 28 .

1.1. Twisted Spin ${ }^{c}$ structures and associated Dirac operators. Let $X$ be a closed oriented Riemannian four-manifold with a Spin ${ }^{c}$ structure $\mathfrak{s}$ on it. The $\operatorname{Spin}^{c}$ structure consists of two Hermitian rank 2 vector bundles $S_{\mathfrak{s}}^{ \pm}$with identified determinant line bundles and a Clifford multiplication

$$
\gamma: \Lambda^{1}\left(T^{*} X\right) \rightarrow \operatorname{Hom}_{\mathbb{C}}\left(S_{\mathfrak{s}}^{+}, S_{\mathfrak{s}}^{-}\right) .
$$

The Clifford map $\gamma$ is, up to a universal constant, an isometry of the cotangent bundle onto a real form inside $\operatorname{Hom}_{\mathbb{C}}\left(S_{\mathfrak{s}}^{+}, S_{\mathfrak{s}}^{-}\right)$which can be specified by the Pauli matrices. We extend $\gamma$ to $\operatorname{End}\left(S_{\mathfrak{s}}^{+} \oplus S_{\mathfrak{s}}^{-}\right)$by $-\gamma^{*}$ on the negative Spinor bundle. 
It then naturally extends to exteriour powers of $T^{*} X$, and in particular its restriction to self-dual two-forms is zero on the negative Spinor bundle, and induces an isomorphism

$$
\gamma: \Lambda_{+}^{2}\left(T^{*} X\right) \stackrel{\cong}{\rightrightarrows} \mathfrak{s u}\left(S_{\mathfrak{s}}^{+}\right) .
$$

Furthermore suppose we are given a Hermitian vector bundle $E$ with determinant line bundle $w=\operatorname{det}(E)$ on $X$. We can then form spinor bundles

$$
W_{\mathfrak{s}, E}^{ \pm}:=S_{\mathfrak{s}}^{ \pm} \otimes E \text {. }
$$

Clifford multiplication extends by tensoring with the identity on $E$. This way we obtain a $S p i n^{c}$ - structure 'twisted' by the hermitian bundle $E$.

Taking the tensor product with the identity on $E$ induces a Clifford multiplication $\gamma: \Lambda^{1}\left(T^{*} X\right) \rightarrow \operatorname{Hom}\left(W_{\mathfrak{s}, E}^{+}, W_{\mathfrak{s}, E}^{-}\right)$. Let's fix a background $\operatorname{Spin}^{c}$ connection $B$ on $\mathfrak{s}$ (that will always stay the same) and suppose we are given a unitary connection $A$ on $E$. By composing the tensor product connection $\nabla_{B} \otimes \nabla_{A}$ with the Clifford multiplication we get a Dirac operator

$$
\not D_{A}^{ \pm}:=\gamma \circ\left(\nabla_{B} \otimes \nabla_{A}\right): \Gamma\left(X ; W_{\mathfrak{s}, E}^{ \pm}\right) \rightarrow \Gamma\left(X ; W_{\mathfrak{s}, E}^{\mp}\right) .
$$

This is a self-adjoint first order elliptic operator. We have oppressed the Spin ${ }^{c}$ connection $B$ from the notation because it will not be a variable in our theory.

1.2. Algebraic preliminaries. We shall now introduce the quadratic map $\mu_{0, \tau}$ : $S^{+} \otimes E \rightarrow \mathfrak{s u}\left(S^{+}\right) \otimes_{\mathbb{R}} \mathfrak{s u}(E)$, defined for a real number $\tau \in[0,1]$, that appears in the non-abelian monopole equations. For $\tau=0$ it will appear in the $P U(n)$ monopole equations, and for $\tau \neq 0$ in the $U(n)$ monopole equations. This map is a natural generalisation of the corresponding map appearing in the situation of $P U(2)$ monopoles [7, 9, 27] as well as the one in the classical (abelian) SeibergWitten equations [14, 29].

The twisted spinor bundles $W_{\mathfrak{s}, E}^{ \pm}$are associated bundles of the fibre product of a $\operatorname{Spin}^{c}$ principal bundle and a $U(n)$-principal bundle on X, with the standard fibre $\mathbb{C}^{2} \otimes \mathbb{C}^{n}$

Let us consider the isomorphism

$$
\begin{aligned}
(p, q): \mathfrak{g l}\left(\mathbb{C}^{n}\right) & \rightarrow \mathfrak{s l}\left(\mathbb{C}^{n}\right) \oplus \mathbb{C} \text { id } \\
a & \mapsto\left(a-\frac{1}{n} \operatorname{tr}(a) \cdot \mathrm{id}, \frac{1}{n} \operatorname{tr}(a) \cdot \mathrm{id}\right) .
\end{aligned}
$$

Both components $p$ and $q$ are orthogonal projections onto their images. Note that $\mathfrak{g l}\left(\mathbb{C}^{2}\right) \otimes \mathfrak{g l}\left(\mathbb{C}^{n}\right)$ and $\mathfrak{g l}\left(\mathbb{C}^{2} \otimes \mathbb{C}^{n}\right)$ are canonically isomorphic. We define the orthogonal projections

$$
\begin{aligned}
& P: \mathfrak{g l}\left(\mathbb{C}^{2} \otimes \mathbb{C}^{n}\right) \rightarrow \mathfrak{s l}\left(\mathbb{C}^{2}\right) \otimes \mathfrak{s l}\left(\mathbb{C}^{n}\right), \\
& Q: \mathfrak{g l}\left(\mathbb{C}^{2} \otimes \mathbb{C}^{n}\right) \rightarrow \mathfrak{s l}\left(\mathbb{C}^{2}\right) \otimes \mathbb{C i d}
\end{aligned}
$$

to be the tensor product ()$_{0} \otimes p$ respectively ()$_{0} \otimes q$, with ()$_{0}$ denoting the trace-free part of the endomorphism of the first factor $\mathbb{C}^{2}$.

For elements $\Psi, \Phi \in \mathbb{C}^{2} \otimes \mathbb{C}^{n}$ we define

$$
\mu_{0, \tau}(\Psi, \Phi):=P\left(\Psi \Phi^{*}\right)+\tau Q\left(\Psi \Phi^{*}\right),
$$

where $\left(\Psi \Phi^{*}\right) \in \mathfrak{g l}\left(\mathbb{C}^{2} \otimes \mathbb{C}^{n}\right)$ is defined to be the endomorphism $\Xi \mapsto \Psi(\Phi, \Xi)$. 
With this notation $\mu_{0,1}(\Psi, \Phi)$ is simply the orthogonal projection of the endomorphism $\Psi \Phi^{*} \in \mathfrak{g l}\left(\mathbb{C}^{2} \otimes \mathbb{C}^{n}\right)$ onto $\mathfrak{s l}\left(\mathbb{C}^{2}\right) \otimes \mathfrak{g l}\left(\mathbb{C}^{n}\right)$. We shall also write $\mu_{0, \tau}(\Psi):=$ $\mu_{0, \tau}(\Psi, \Psi)$ for the associated quadratic map. In the case $n=1$ the map $\mu_{0,1}(\Psi)$ is the quadratic map in the spinor usually occuring in the Seiberg-Witten equations 29] [14. The proof of the following proposition can be found in [?].

Proposition 1.1. Suppose $n>1$. Then the quadratic map $\mu_{0, \tau}$ is uniformly proper. In other words, there is a positive constant $c>0$ such that

$$
\left|\mu_{0, \tau}(\Psi)\right| \geq c|\Psi|^{2}
$$

As a consequence we have the formula

$$
\left(\mu_{0, \tau}(\Psi) \Psi, \Psi\right) \geq c^{2}|\Psi|^{4}
$$

whenever $\tau \geq 0$. Furthermore, suppose $n \geq 2$ or $\tau \neq 0$. Then the bilinear map $\mu_{0, \tau}$ is 'without zero-divisors' in the following sense: If $\mu_{0, \tau}(\Psi, \Phi)=0$, then either $\Psi=0$ or $\Phi=0$.

Proof: Obviously we have $\left|\mu_{0, \tau}(\Psi)\right| \geq\left|\mu_{0,0}(\Psi)\right|$, so for the first assertion it will be enough to consider $\mu_{0,0}$ alone. We will show that $\mu_{0,0}(\Psi)=0$ implies $\Psi=0$. Because $\mu_{0,0}$ is quadratic and the unit sphere inside $\mathbb{C}^{2} \otimes \mathbb{C}^{n}$ is compact we then get the claimed uniform properness-inequality (2).

We shall use the canonical isomorphism $\mathbb{C}^{2} \otimes \mathbb{C}^{n} \cong \mathbb{C}^{n} \oplus \mathbb{C}^{n}$, which permits to write a general element

as

$$
\Psi=\left(\begin{array}{l}
1 \\
0
\end{array}\right) \otimes \alpha+\left(\begin{array}{l}
0 \\
1
\end{array}\right) \otimes \beta
$$

We then have

$$
\Psi=\left(\begin{array}{l}
\alpha \\
\beta
\end{array}\right) .
$$

$$
\begin{aligned}
\mu_{0,0}(\Psi) & =P\left(\Psi \Psi^{*}\right) \\
& =P\left(\left(\begin{array}{l}
\alpha \\
\beta
\end{array}\right)\left(\alpha^{*} \beta^{*}\right)\right) \\
& =P\left(\begin{array}{ll}
\alpha \alpha^{*} & \alpha \beta^{*} \\
\beta \alpha^{*} & \beta \beta^{*}
\end{array}\right) \\
& =\left(\begin{array}{cc}
\frac{1}{2}\left(\alpha \alpha^{*}-\beta \beta^{*}\right)_{0} & \left(\alpha \beta^{*}\right)_{0} \\
\left(\beta \alpha^{*}\right)_{0} & \frac{1}{2}\left(\beta \beta^{*}-\alpha \alpha^{*}\right)_{0}
\end{array}\right) .
\end{aligned}
$$

In particular, if $\mu_{0,0}(\Psi)=0$, then we have $\left(\alpha \beta^{*}\right)_{0}=0$.

Lemma 1.2. The equation $\left(\alpha \beta^{*}\right)_{0}=0$ implies that $\alpha=0$ or $\beta=0$. In other words, the bilinear map $(\alpha, \beta) \rightarrow\left(\alpha \beta^{*}\right)_{0}$ is without zero-divisors (here $n \geq 2$ is implicitely understood).

Proof of Lemma 1.2: Write the elements $\alpha$ and $\beta$ as

$$
\alpha=\left(\alpha_{i}\right)_{i=1}^{n}, \quad \beta=\left(\beta_{i}\right)_{i=1}^{n} .
$$

Then the equation $\left(\alpha \beta^{*}\right)_{0}=0$ reads in matrix-form

$$
\left(\begin{array}{ccc}
\alpha_{1} \overline{\beta_{1}}-\frac{1}{n} \sum \alpha_{i} \overline{\beta_{i}} & \ldots & \alpha_{1} \overline{\beta_{n}} \\
\vdots & \ddots & \vdots \\
\alpha_{n} \overline{\beta_{1}} & \ldots & \alpha_{n} \overline{\beta_{n}}-\frac{1}{n} \sum \alpha_{i} \overline{\beta_{i}}
\end{array}\right)=0 .
$$


Suppose $\beta \neq 0$, for instance $\beta_{j} \neq 0$. Then the $j^{\text {th }}$ column implies that $\alpha_{i}=0$ for all $i \neq j$. Thus the $j^{\text {th }}$ element in the $j^{\text {th }}$ column simplifies,

$$
\alpha_{j} \overline{\beta_{j}}-\frac{1}{n} \sum_{i=1}^{n} \alpha_{i} \overline{\beta_{i}}=\left(1-\frac{1}{n}\right) \alpha_{j} \overline{\beta_{j}} .
$$

Therefore we have $\alpha_{j}=0$ as well, so that we have $\alpha=0$. The case $\alpha \neq 0$ is analogous.

Returning to the problem

$$
\left(\begin{array}{cc}
\frac{1}{2}\left(\alpha \alpha^{*}-\beta \beta^{*}\right)_{0} & \left(\alpha \beta^{*}\right)_{0} \\
\left(\beta \alpha^{*}\right)_{0} & \frac{1}{2}\left(\beta \beta^{*}-\alpha \alpha^{*}\right)_{0}
\end{array}\right)=0,
$$

we see that the lemma gives $\alpha=0$ or $\beta=0$. Suppose, without loss of generality, that the first is the case. Then we are left with $\left(\beta \beta^{*}\right)_{0}=0$. Now again with lemma 1.2 we see that this also implies $\beta=0$. Therefore

$$
\Psi=\left(\begin{array}{l}
\alpha \\
\beta
\end{array}\right)=0 .
$$

The second assertion now follows from the first, remembering that $P$ and $Q$ are both orthogonal projections. For non-negative $\tau$ we have the inequality

$$
\begin{aligned}
\left(\mu_{0, \tau}(\Psi) \Psi, \Psi\right) & =\left(P\left(\Psi \Psi^{*}\right) \Psi, \Psi\right)+\tau\left(Q\left(\Psi \Psi^{*}\right) \Psi, \Psi\right) \\
& =\left(P\left(\Psi \Psi^{*}\right), \Psi \Psi^{*}\right)+\tau\left(Q\left(\Psi \Psi^{*}\right), \Psi \Psi^{*}\right) \\
& =\left(P\left(\Psi \Psi^{*}\right), P\left(\Psi \Psi^{*}\right)\right)+\tau\left(Q\left(\Psi \Psi^{*}\right), Q\left(\Psi \Psi^{*}\right)\right) \\
& \geq\left|\mu_{0,0}(\Psi)\right|^{2} \\
& \geq c^{2}|\Psi|^{4} .
\end{aligned}
$$

Similarly, one shows the claimed property about the 'zero-divisors'.

Because of the equivariance property of the map $\mu_{0, \tau}$ we get in a straightforward way corresponding maps between bundles, giving rise to

$$
\mu_{0, \tau}: W_{\mathfrak{s}, E}^{ \pm} \times W_{\mathfrak{s}, E}^{ \pm} \rightarrow \mathfrak{s l}\left(S_{\mathfrak{s}}^{ \pm}\right) \otimes \mathbb{C} \mathfrak{g l}(E),
$$

respectively, for the quadratic map,

$$
\mu_{0, \tau}: W_{\mathfrak{s}, E}^{ \pm} \rightarrow \mathfrak{s u}\left(S_{\mathfrak{s}}^{ \pm}\right) \otimes_{\mathbb{R}} \mathfrak{u}(E),
$$

or

$$
\mu_{0,0}: W_{\mathfrak{s}, E}^{ \pm} \rightarrow \mathfrak{s u}\left(S_{\mathfrak{s}}^{ \pm}\right) \otimes_{\mathbb{R}} \mathfrak{s u}(E)
$$

if we put $\tau=0$.

These maps on the bundle level satisfy the corresponding statement in the above proposition with the same constant $c$.

Definition 1.3. If we wish to make precise to which Hermitian bundle E we refer we shall denote the corresponding bundle as an upper-script $\mu_{0, \tau}^{E}$. 
1.3. $\mathbf{P U}(\mathbf{N})$-monopole equations. In this manuscript we shall not be concerned about the analytical properties of the moduli spaces involved, such as the question of transversality. Therefore, as a matter of convenience, we shall work throughout with spaces of smooth (infinitely differentiable) sections, connections... It will be clear how to formulate a corresponding theory with Sobolev completions as is usually done in gauge theory.

Let $\theta$ be a fixed smooth unitary connection in the determinant line bundle $w$. We shall denote by $\mathscr{A}_{\theta}(E)$ the space of smooth unitary connections on $E$ which induce the fixed connection $\theta$ in $w$. This is an affine space modelled on $\Omega^{1}(X ; \mathfrak{s u}(E))$. Here $\mathfrak{s u}(E)$ denotes the bundle of skew-adjoint trace-free endomorphisms of $E$. Furthermore $\Gamma\left(X ; W_{\mathfrak{s}, E}^{+}\right)$denotes the space of smooth sections of the spinor bundle $W_{\mathfrak{s}, E}^{+}$. We define our configuration space to be

$$
\mathscr{C}_{\mathfrak{s}, E, \theta}:=\Gamma\left(X ; W_{\mathfrak{s}, E}^{+}\right) \times \mathscr{A}_{\theta}(E) .
$$

We denote by $\mathscr{G}^{0}$ the group of unitary automorphisms of $E$ with determinant 1 ; it will be the 'gauge group' of our moduli problem. It acts in a canoncial way on sections of the spinor bundles, and as $\left(u, \nabla_{A}\right) \mapsto u \nabla_{A} u^{-1}$ on the connections, where $u$ is a gauge transformation and $\nabla_{A}$ a unitary connection. In particular it lets the induced connection in the determinant line bundle $w$ fixed. The set $\mathscr{B}_{\mathfrak{s}, E, \theta}$ is defined to be the configuration space up to gauge, that is the quotient space $\mathscr{C}_{\mathfrak{s}, E, \theta} / \mathscr{G}^{0}$.

We are now able to write down the $\mathrm{PU}(\mathrm{N})$-monopole equations associated to the data $(\mathfrak{s}, E)$ consisting of a $\operatorname{Spin}^{c}$-structure $\mathfrak{s}$ and a unitary bundle $E$ of rank $N$ on $X$. These equations read as follows:

$$
\begin{aligned}
\not_{A}^{+} \Psi & =0 \\
\gamma\left(\left(F_{A}^{+}\right)_{0}\right)-\mu_{0,0}(\Psi) & =0 .
\end{aligned}
$$

Here $\left(F_{A}\right)_{0} \in \Omega^{2}(X ; \mathfrak{s u}(E))$ denotes the trace-free part of the curvature $F_{A} \in$ $\Omega^{2}(X ; \mathfrak{u}(E))$.

The left hand side of the above equations can be seen as a map $\mathscr{F}$ of the configuration space $\mathscr{C}=\Gamma\left(X, W_{\mathfrak{s}, E}^{+}\right) \times \mathscr{A}_{\theta}\left(P_{E}\right)$ to the space $\Gamma\left(X, W_{\mathfrak{s}, E}^{-}\right) \times \Gamma\left(X, \mathfrak{s u}\left(S_{\mathfrak{s}}^{+}\right) \otimes\right.$ $\mathfrak{s u}(E))$. As such it satisfies the equivariance property

$$
\mathscr{F}(u \cdot(\Psi, A))=\left(u \times \operatorname{ad}_{u}\right)(\mathscr{F}(\Psi, A)) .
$$

Therefore it is sensible to define:

Definition 1.4. The moduli space $M_{\mathfrak{s}, E, \theta}$ of $P U(N)$ monopoles is defined to be the solution set of the equations (4) associated to the data $(\mathfrak{s}, E)$ modulo the gauge-group $\mathscr{G}^{0}$ :

$$
M_{\mathfrak{s}, E, \theta}:=\left\{[\Psi, A] \in \mathscr{B}_{\mathfrak{s}, E, \theta} \mid \mathscr{F}(\Psi, A)=0\right\} .
$$

There is an elliptic deformation complex associated to a solution $(\Psi, A)$ of the $P U(N)$ monopole equations. Its index equals minus the 'expected dimension' of the moduli space. This expected dimension is computed by the Atiyah-Singer index theorem and is given by the index of the deformation operator $\delta_{A}=\left(-d_{A}^{*} \oplus d_{A}^{+}\right)$ of the $P U(N)$ instantons 13 plus twice the complex index of the twisted Dirac 
operator,

$$
\begin{aligned}
\operatorname{ex}-\operatorname{dim}\left(M_{\mathfrak{s}, E, \theta}\right)= & \operatorname{ind}\left(\delta_{A}\right) \oplus 2 \operatorname{ind}_{\mathbb{C}}\left(\not_{A}^{+}\right) \\
= & -2\left\langle p_{1}(\mathfrak{s u}(E)),[X]\right\rangle-\left(N^{2}-1\right)\left(b_{2}^{+}(X)-b_{1}(X)+1\right) \\
& +\left\langle\operatorname{ch}(E) e^{\frac{1}{2} c_{1}\left(S_{\mathfrak{s}}^{+}\right)} \hat{\mathrm{A}}(T X),[X]\right\rangle
\end{aligned}
$$

where $p_{1}(\mathfrak{s u}(E))$ denotes the first Pontryagin class of the bundle $\mathfrak{s u}(E), b_{2}^{+}(X)$ the maximal dimension of a subspace of $H_{2}(X ; \mathbb{R})$ on which the intersection form of $X$ is positive definite, and $\hat{\mathrm{A}}(T X)=1-\frac{1}{24} p_{1}(T X)$ is the $\hat{\mathrm{A}}$-genus of the four-manifold $X$.

1.4. Uniform bound, compactification. The moduli space $M_{\mathfrak{s}, E, \theta}$ turns out to be non-compact in general, but possesses a canonical compactification very analogue to the Uhlenbeck-compactification of instanton moduli spaces [5]. The main reason is that there is a uniform $C^{0}$ bound on the spinor part $\Psi$ of $P U(N)$ monopoles $[\Psi, A] \in M_{\mathfrak{s}, E, \theta}$. Knowing this, the compactification is fairly standard [5], 27], [8], and therefore we will keep our exposition very brief on this point. An outline for the $P U(N)$ case can also be found in [31.

The $C^{0}$ bound is derived similarly to classical Seiberg-Witten theory [14 from the Weitzenböck formula for the Dirac-operator $D_{A}$ by making also use of the above Proposition 1.1

Proposition 1.5. There are constants $c, K \in \mathbb{R}$, with $c>0$, such that for any monopole $[\Psi, A] \in M_{\mathfrak{s}, E}$ we have a $C^{0}$ bound:

$$
\max |\Psi|^{2} \leq \max \left\{0, K / c^{2}\right\} .
$$

Here the constant $K$ depends on the Riemannian metric, the fixed background Spin ${ }^{c}$ connection whereas the constant $c$ is universal.

Let $\mathfrak{s}$ be a $\operatorname{Spin}^{c}$-structure on $X$ and let $E \rightarrow X$ be a unitary bundle on $X$. We denote by $E_{-k}$ a bundle which has first Chern class $c_{1}\left(E_{-k}\right)=c_{1}(E)$ and whose second Chern class satisfies

$$
\left\langle c_{2}\left(E_{-k}\right),[X]\right\rangle=\left\langle c_{2}(E),[X]\right\rangle-k .
$$

On a four-manifold such a bundle is unique up to isomorphism.

Definition 1.6. An ideal $P U(N)$ monopole associated to the data $(\mathfrak{s}, E, \theta)$ is given by a pair $([\Psi, A], \mathbf{x})$, where $[\Psi, A] \in M_{\mathfrak{s}, E_{-k}, \theta}$ is a $P U(N)$ monopole associated to $\left(\mathfrak{s}, E_{-k}\right)$ monopole, and $\mathbf{x}$ is an element of the $k$-th symmetric power $\operatorname{Sym}^{k}(X)$ of $X$ (that is, an unordered set of $k$ points in $X, \mathbf{x}=\left\{x_{1}, \ldots, x_{k}\right\}$ ). The curvature density of $([\Psi, A], \mathbf{x})$ is defined to be the measure

$$
\left|F_{A}\right|^{2}+8 \pi^{2} \sum_{x_{i} \in \mathbf{x}} \delta_{x_{i}} .
$$

The set of ideal monopoles associated to the data $(\mathfrak{s}, E, \theta)$ is

$$
I M_{\mathfrak{s}, E, \theta}:=\coprod_{k \geq 0} M_{\mathfrak{s}, E_{-k}, \theta} \times \operatorname{Sym}^{k}(X),
$$


which is endowed with a convenient topology [5], 27]. Rougly speaking, in this topology a sequence in the main stratum $\left[\Psi_{n}, A_{n}\right]$ converges to a point $([\Phi, B], \mathbf{x}) \in$ $M_{\mathfrak{s}, E_{-k}, \theta} \times \operatorname{Sym}^{k}(X)$ if the sequence of measures $\left|F_{A_{n}}\right|^{2}$ vol converges to the measure given by $\left|F_{B}\right|^{2} \mathrm{vol}+8 \pi^{2} \sum_{x_{i} \in \mathbf{x}} \delta_{x_{i}}$, and if $\Psi_{n}$ converges to $\Phi$ in the complement of $\mathbf{x}$ in $X$. The main result is then:

Theorem 1.7. (Compactness-Theorem) The closure of $M_{\mathfrak{s}, E, \theta}$ inside the space of ideal monopoles $I M_{\mathfrak{s}, E, \theta}$ is compact.

1.5. U(n) monopoles. We shall denote by $\mathscr{A}(E)$ the space of smooth unitary connections on $E$ which is an affine space modelled on $\Omega^{1}(X ; \mathfrak{u}(E))$. Here $\mathfrak{u}(E)$ denotes the bundle of skew-adjoint endomorphisms of $E$. We define our configuration of $U(n)$ monopoles to be the space

$$
\mathscr{C}_{\mathfrak{s}, E}:=\Gamma\left(X ; W_{\mathfrak{s}, E}^{+}\right) \times \mathscr{A}(E) .
$$

We denote by $\mathscr{G}$ the group of unitary automorphisms of $E$ (and not just of determinant 1, as for $P U(N)$ monopoles); it is the 'gauge group' of our problem. The space $\mathscr{B}_{\mathfrak{s}, E}$ is defined to be the configuration space up to gauge, i.e. the quotient space $\mathscr{C}_{\mathfrak{s}, E} / \mathscr{G}$.

For a configuration $(\Psi, A) \in \mathscr{C}_{\mathfrak{s}, E}$ the $U(n)$-monopole equations with parameter $\tau \in[0,1]$ and perturbation $\eta \in \Omega_{+}^{2}(X ; i \mathbb{R})$ read

$$
\begin{aligned}
\not D_{A}^{+} \Psi & =0 \\
\gamma\left(F_{A}^{+}\right)-\mu_{0, \tau}(\Psi) & =\gamma(\eta) \text { id } .
\end{aligned}
$$

Here $F_{A}$ designs the curvature of the connection $A$ and $F_{A}^{+}$its selfdual part.

Remark. Notice that the curvature equation of (8) splits according to the Lie algebra decomposition $\mathfrak{u}(n)=\mathfrak{s u}(n) \oplus i \mathbb{R}$ into two equations:

$$
\begin{aligned}
\gamma\left(\left(F_{A}^{+}\right)_{0}\right)-\mu_{0,0}(\Psi) & =0 \\
\gamma\left(F_{\operatorname{det}(A)}^{+}\right)-\operatorname{tr} \mu_{0,1}(\Psi) & =n \gamma(\eta) .
\end{aligned}
$$

Therefore we see that the $U(n)$ monopole equation is in fact a coupled equation in the following sense: A solution $(A, \Psi)$ is a $P U(n)$ monopole associated to the 'parameter' $\theta=\operatorname{det}(A)$, so that $(\operatorname{det}(A), \Psi)$ solves some sort of 'perturbed $U(1)$ anti-self-duality equation'

As above, the left hand side of the above equations can be seen as a map $\mathscr{F}_{\tau}$ from the configuration space $\mathscr{C}_{\mathfrak{s}, E}$ to the space $\Gamma\left(X ; W_{\mathfrak{s}, E}^{-}\right) \times \Gamma\left(X ; \mathfrak{s u}\left(S_{\mathfrak{s}}^{+}\right) \otimes \mathfrak{u}(E)\right)$. This map is equivariant with respect to the gauge group $\mathscr{G}$. The moduli space is then defined to be the space of solutions to the monopole equations modulo gauge:

$$
M_{\mathfrak{s}, E}(\tau, \eta):=\left\{[\Psi, A] \in \mathscr{B}_{\mathfrak{s}, E} \mid \mathscr{F}_{\tau}(\Psi, A)=(0, \gamma(\eta))\right\} .
$$

Again, there is an elliptic deformation complex associated to a solution these $U(n)$ monopole equations. The 'expected dimension' of the moduli space is given by the following formula, compare formula (9) above:

$$
\begin{aligned}
\operatorname{ex}-\operatorname{dim}\left(M_{\mathfrak{s}, E}(\tau, \eta)\right)= & -2\left\langle p_{1}(\mathfrak{s u}(E)),[X]\right\rangle-n^{2}\left(b_{2}^{+}(X)-b_{1}(X)+1\right) \\
& +\left\langle\operatorname{ch}(E) e^{\frac{1}{2} c_{1}\left(S_{\mathfrak{s}}^{+}\right)} \hat{\mathrm{A}}(T X),[X]\right\rangle .
\end{aligned}
$$

There is also an Uhlenbeck-compactification of the moduli space $M_{\mathfrak{s}, E}(\tau, \eta)$ that is stated identically to that of the $P U(N)$ monopole moduli space $M_{\mathfrak{s}, E, \theta}$ in section 
1.4 above. In particular, the lower strata still contain bundles $E_{-k}$ with the same first Chern class as $E$.

\section{The CIRCLE-ACTION AND ITS FIXED-POINT SET, RELATIONS TO $\mathrm{U}(\mathrm{N})$-MONOPOLES}

There is a circle-action on the configuration space modulo gauge $\mathscr{B}_{\mathfrak{s}, E, \theta}$ which is induced by multiplying spinors with complex numbers of unit norm. The fixed-point set of this circle-action obviously contains elements with zero spinor component, and the other elements are those which have a connection that splits up into the direct sum of two connections on proper subbundles on $E$ and which have the spinor component being a section of one of these subbundles. The latter fixedpoint loci are naturally labelled by isomorphism classes of proper subbundles of $E$. We shall describe a way of parametrising these fixed point loci by picking a representative vector bundle for each isomorphism class. Next we restrict our considerations to the intersection of the fixed-point set with the moduli space of $P U(N)$ monopoles: fixed-points with vanishing spinor are then simply anti-selfdual $P U(N)$ - connections in $E$, whereas the other fixed-point loci are fibrations of moduli spaces of $P U(n)$-instantons in a summand $F$ of $E$ of rank $n$ over moduli spaces of $U(N-n)$-monopoles in the complement $F^{\perp}$ of $F$ in $E$.

Remark. In this section both configuration spaces $\mathscr{C}_{\mathfrak{s}, E, \theta}$ and moduli spaces $M_{\mathfrak{s}, E, \theta}$ of $P U(N)$ monopoles as configuration spaces $\mathscr{C}_{\mathfrak{s}, F}$ and moduli spaces $M_{\mathfrak{s}, F}$ of $U(n)$ monopoles appear. We remind the reader that our distinction in the notation is by the fixed connection $\theta$ in the determinant line bundle in the $P U(N)$ situation.

2.1. Reductions and stabilisers of connections under the gauge group. Here we study the stabilisers of connections $A \in \mathscr{A}_{\theta}\left(P_{E}\right)$ under the action of the gauge group $\mathscr{G}^{0}$.

An element $u$ of the gauge group $\mathscr{G}^{0}$ acts on the set of connections $A$ in $\mathscr{A}_{\theta}\left(P_{E}\right)$ by the formula

$$
u(A)=A-\left(d_{A} u\right) u^{-1},
$$

where we consider $u$ as section of the vector bundle $\mathfrak{g l}\left(P_{E}\right)$. The stabiliser of the connection $A$ inside the gauge group $G$ is the group of automorphisms which preserve $A$ :

$$
\begin{aligned}
\Gamma(A) & =\left\{u \in \mathscr{G}^{0} \mid u(A)=A\right\} \\
& =\left\{u \in \mathscr{G}^{0} \mid d_{A} u=0\right\}
\end{aligned}
$$

This group $\Gamma(A)$ is a finite-dimensional compact Lie-group and can be seen as a closed Lie subgroup of $\operatorname{Aut}\left(E_{x}\right) \cong U(N)$ for any point $x \in X$ if $X$ ix connected.

Note that the stabiliser always contains the centre $Z(G)$ of the structure group $G, \Gamma(A) \supseteq Z(G)$. In our case, the centre injects as

$$
Z(S U(N))=\left\{\lambda \operatorname{id}_{E} \mid \lambda^{N}=1\right\}
$$

Definition 2.1. The connection $A \in \mathscr{A}_{\theta}\left(P_{E}\right)$ is called reducible if the stabiliser $\Gamma(A)$ is different from the centre $Z(S U(N))$.

Suppose now that $A$ is reducible and that $d_{A} u=0$. We recall a standard result for normal endomorphisms of a Hermitian vector-bundle. An endomorphism $u$ is 
called normal if $u u^{*}=u^{*} u$, where $u^{*}$ is the adjoint endomorphism with respect to the Hermitian structure. The following lemma is easy to prove:

Lemma 2.2. Suppose the normal endomorphism $u \in \operatorname{End}(E)$ is A-parallel, $d_{A} u=$ 0 . Then its spectrum is constant and there is a A-parallel decomposition of $E$ into subbundles

$$
E=\bigoplus_{\lambda \in \operatorname{Spec}(u)} E_{\lambda} .
$$

Each summand $E_{\lambda}$ is $u$-invariant, and we have $\left.u\right|_{E_{\lambda}}=\lambda i d_{E_{\lambda}}$. In other words, the $E_{\lambda}$ are eigen-bundles of the endomorphism $u$.

As a corollary one obtains that a connection $A$ is reducible if and only if there is a proper subbundle $F$ of $E$ which is $A$ - parallel. For, the latter condition clearly implies that $A$ is reducible in our definition. On the other hand, if the stabiliser $\Gamma(A)$ is strictly bigger than the centre $Z(S U(N))$, then by the preceding lemma there must be a $A$-parallel automorphism $u \in \mathscr{G}^{0}$ which admits an eigenvalue which is not an $N^{\text {th }}$ root of one, and therefore there must be such an $A$-parallel subbundle.

2.2. Stabiliser of a configuration under the gauge group. We define the stabiliser $\Gamma(\Psi, A)$ of a configuration $(\Psi, A) \in \mathscr{C}_{\mathfrak{s}, E}$ to be the set

$$
\begin{aligned}
\Gamma((\Psi, A)) & =\left\{u \in \mathscr{G}^{0} \mid u(\Psi, A)=(\Psi, A)\right\} \\
& =\left\{u \in \mathscr{G}^{0} \mid u(\Psi)=\Psi, \quad d_{A} u=0\right\} .
\end{aligned}
$$

Definition 2.3. The subset $\mathscr{C}_{\mathfrak{s}, E, \theta}^{*} \subseteq \mathscr{C}_{\mathfrak{s}, E, \theta}$ (respectively $\mathscr{C}_{\mathfrak{s}, E, \theta}^{* *}$ ) is defined to be the set of configurations $(\Psi, A) \in \mathscr{C}_{\mathfrak{s}, E, \theta}$ which has zero-dimensional stabiliser (respectively trivial stabiliser). The subset $\mathscr{B}_{\mathfrak{s}, E, \theta}^{*} \subseteq \mathscr{B}_{\mathfrak{s}, E, \theta}$ is defined to be the subset $\mathscr{C}_{\mathfrak{s}, E, \theta}^{*} / \mathscr{G}^{0} \subseteq \mathscr{B}_{\mathfrak{s}, E, \theta}$. The subset $\mathscr{B}_{\mathfrak{s}, E, \theta}^{* *}$ is defined correspondingly.

It is easy to see that the stabiliser of a configuration $(\Psi, A)$, with $\Psi$ non-vanishing and the connection $A$ irreducible, is trivial. Furthermore, the configurations $(\Psi, A)$ with $\Psi \equiv 0$ and irreducible connection part $A$ have stabilisers which are the finite group $Z(S U(N))$. These claims, as well as the following Proposition, follow easily from Lemma (2.2) in the section of reducible connections above.

Proposition 2.4. Suppose we have given a configuration $(\Psi, A)$ with non-vanishing spinor $\Psi$, and the connection of the form $A=A_{1} \oplus A_{2}$ according to a A-parallel decomposition $E=F \oplus F^{\perp}$, with both $A_{1}$ and $A_{2}$ irreducible. Then its stabiliser is a finite (and thus zero-dimensional) group.

2.3. The circle action. We are given an $S^{1}$-action on the configuration space $\mathscr{C}_{\mathfrak{s}, E}$ given by the simple formula

$$
\begin{aligned}
S^{1} \times \mathscr{C}_{\mathfrak{s}, E} & \rightarrow \mathscr{C}_{\mathfrak{s}, E} \\
(z,(\Psi, A)) & \mapsto(z \Psi, A)
\end{aligned}
$$

Now as this action commutes with the action of the gauge group $\mathscr{G}^{0}$, we see that we get a well-defined action on the quotient,

$$
S^{1} \times \mathscr{B}_{\mathfrak{s}, E, \theta} \rightarrow \mathscr{B}_{\mathfrak{s}, E, \theta}
$$


The action is not effective. In fact if $z^{N}=1$ there is always a gauge-transformation $u$ with $u(\Psi, A)=(z \Psi, A)$, that is $[\Psi, A]=[z \Psi, A]$, because $Z(S U(N)) \subseteq \Gamma(A)$. Therefore we define

$$
r(z,[\Psi, A]):=\left[z^{1 / N} \Psi, A\right] .
$$

In this formula $z^{1 / N}$ is any $N^{\text {th }}$ root of 1 , the equvialence class $\left[z^{1 / N} \Psi, A\right]$ does not depend on the particular choice.

Remark. Suppose we had chosen as gauge-group $\mathscr{G}^{0}$ the group of unitary bundleautomorphisms of $E$ which fix the connection $\theta$ only, that is, the larger group of unitary automorphisms with constant determinant. Then the same action on $\mathscr{C}_{\mathfrak{s}, E, \theta}$ would have introduced the trivial $S^{1}$-action on $\mathscr{B}_{\mathfrak{s}, E, \theta}$. This justifies our choice of the gauge group $\mathscr{G}^{0}$ as $\Gamma(S U(E))$.

Lemma 2.5. Suppose there exists some $z_{0} \in S^{1}$ with $z_{0} \neq 1$ such that $\left[z_{0}^{1 / N} \Psi, A\right]=$ $[\Psi, A]$. Then for any $z \in S^{1}$ we have

$$
[z \Psi, A]=[\Psi, A] .
$$

Thus an element $[\Psi, A]$ is a fixed-point of the circle-action if and only if it is left fixed by some non-trivial element $z_{0} \in S^{1}$ under the action $r$.

The proof, using Lemma 2.2, is left to the reader.

Using the above Lemma 2.2 again we deduce the following simple criterion for fixed-points under the action $r$ above:

Proposition 2.6. A configuration up to gauge $[\Psi, A] \in \mathscr{B}_{\mathfrak{s}, E, \theta}$ is contained in the fixed point set of the action $r$ if and only if for some (or equivalently, for any) representative $(\Psi, A)$ we have one of the following (possibly both):

(1) There is a non-trivial A-parallel orthogonal decomoposition $E=\oplus E_{i}$ and the spinor is a section of one of $S_{\mathfrak{s}}^{+} \otimes E_{i}$

(2) The spinor vanishes $\Psi \equiv 0$.

Further down we will see that if we impose in addition the monopole equations the spinor component of a fixed point $[\Psi, A]$ will automatically lie in a proper summand $S^{+} \otimes E_{i}$ as soon as the connection $A$ is reducible.

2.4. The $S^{1}$-fixed point set inside the configuration space modulo gauge, and parametrisations. First we will describe the fixed-point set of the $S^{1}$-action inside $\mathscr{B}_{\mathfrak{s}, E, \theta}$. In the above Proposition 2.6] we saw that these are related to proper subbundles of $E$. However, two subbundles which are mapped into each other by gauge transformations, i.e. automorphisms of E, should be considered equivalent. This equivalence of subbundles might be called 'ambiently isomorphic', but it is easy to see that two subbundles of $E$ are ambiently isomorphic if and only if they are isomorphic as abstract bundles. This even holds for prescribed determinant. For the further work, especially for describing the intersection of the fixed point set with the moduli space, it turns out useful to fix representatives $F \in[F]$ for each such isomorphism class. This yields to a 'parametrisation' of each component of the fixed point set which is determined by the isomorphism class $[F]$. 
Definition 2.7. Let $F$ be a proper summand of the unitary bundle $E$. We define the set $\mathscr{B}_{\mathfrak{s}, E, \theta}^{[F]}$ to be the set of all elements $[\Psi, A] \in \mathscr{B}_{\mathfrak{s}, E, \theta}$ such that for some representative $(\Psi, A)$ there exists a A-parallel decomposition $E=F \oplus F^{\perp}$ with $\Psi \in \Gamma\left(X, S_{\mathfrak{s}}^{+} \otimes F\right)$.

By Proposition 2.6 the space $\mathscr{B}_{\mathfrak{s}, E, \theta}^{[F]}$ is contained in the $S^{1}$-fixed point set $\mathscr{B}_{\mathfrak{s}, E, \theta}^{S^{1}}$. Another subset of the fixed point set is given by the space $\mathscr{B}_{\mathfrak{s}, E}^{\equiv 0}$ defined as the subspace of elements $[\Psi, A] \in \mathscr{B}_{\mathfrak{s}, E, \theta}$ with vanishing spinor component, $\Psi \equiv 0$.

The above Proposition 2.6 gives then the following description of the fixed point set of the $S^{1}$ - action $r$ :

Proposition 2.8. The $S^{1}$-fixed point set $\mathscr{B}_{\mathfrak{s}, E, \theta}^{S^{1}}$ is given as the union

$$
\left(\bigcup_{[F] \subseteq E} \mathscr{B}_{\mathfrak{s}, E, \theta}^{[F]}\right) \bigcup \mathscr{B}_{\mathfrak{s}, E, \theta}^{\equiv 0} .
$$

Here the first union is taken over all isomorphism classes of proper subbundles of E.

We should point out as well that the different components $\mathscr{B}_{\mathfrak{s}, E, \theta}^{[F]}$ may a priori intersect each other or the fixed-point component of vanishing spinor $\mathscr{B}_{\mathfrak{s}, E, \theta}^{\equiv 0}$. We would also like to remark that for rank strictly higher than 2 we may always have infinitely many such isomorphism classes of proper subbundles $[F]$ of $E$, even for definite intersection form.

In order to have a convenient description of the set $\mathscr{B}_{\mathfrak{s}, E, \theta}^{[F]}$ it seems natural to fix an actual proper subbundle $F$ for each isomorphism class $[F]$. Hence the following definition:

Definition 2.9. We define the configuration space relative to the splitting $E=$ $F \oplus F^{\perp}$ as the following set:

$$
\begin{aligned}
\mathscr{C}_{\mathfrak{s}, E, \theta}^{F \oplus F^{\perp}}:= & \left\{\left(\Psi, A_{1}, A_{2}\right) \in \Gamma\left(S_{\mathfrak{s}}^{+} \otimes F\right) \times \mathscr{A}(F) \times \mathscr{A}\left(F^{\perp}\right) \mid\right. \\
& \left.\operatorname{det}\left(A_{1}\right) \otimes \operatorname{det}\left(A_{2}\right)=\theta\right\}
\end{aligned}
$$

Correspondingly, the group of unitary automorphisms with determinant 1 respecting the splitting $E=F \oplus F^{\perp}$ is defined to be

$$
\mathscr{G}_{F \oplus F^{\perp}}^{0}:=\left\{\left(u_{1}, u_{2}\right) \in \Gamma(U(F)) \times \Gamma\left(U\left(F^{\perp}\right)\right) \mid \operatorname{det}\left(u_{1}\right) \cdot \operatorname{det}\left(u_{2}\right)=1\right\}
$$

As usually, we denote the quotient by:

$$
\mathscr{B}_{\mathfrak{s}, E, \theta}^{F \oplus F^{\perp}}:=\mathscr{C}_{\mathfrak{s}, E, \theta}^{F \oplus F^{\perp}} / \mathscr{G}_{F \oplus F^{\perp}}^{0} .
$$

It is then easy to see that this yields a well-defined map

$$
\begin{aligned}
i_{F}: \mathscr{B}_{\mathfrak{s}, E, \theta}^{F \oplus F^{\perp}} \rightarrow \mathscr{B}_{\mathfrak{s}, E, \theta}^{[F]} & \\
& {\left[\Psi, A_{1}, A_{2}\right] \mapsto\left[\Psi, A_{1} \oplus A_{2}\right] . }
\end{aligned}
$$

This map is easily seen to be always surjective. However, it fails to be injective in general. Nonetheless, on a dense subset of $\mathscr{B}_{\mathfrak{s}, E}^{F \oplus F^{\perp}}$ it is, as we shall show next. We will think of the map $i_{F}$ as a 'parametrisation' of the fixed-point set component $\mathscr{B}_{\mathfrak{s}, E}^{[F]}$. 
As before, let us denote by $\mathscr{B}_{\mathfrak{s}, E}^{* F} \oplus F^{\perp}$ and by $\mathscr{B}_{\mathfrak{s}, E}^{*[F]}$ the configurations which have finite-dimensional stabiliser in their groups $\mathscr{G}^{0}$ respectively $\mathscr{G}_{F \oplus F^{\perp}}^{0}$. Further, we denote by $\mathscr{B}_{\mathfrak{s}, E, \theta}^{*, i r r} F \oplus F^{\perp}$ the subset of $\mathscr{B}_{\mathfrak{s}, E, \theta}^{*} F \oplus F^{\perp}$ consisting of elements [ $\left.\Psi, A_{1}, A_{2}\right]$ with non-vanishing spinor, $\Psi \neq 0$, and both connections $A_{1}$ and $A_{2}$ irreduible. By the way, $\left(\Psi, A_{1}, A_{2}\right)$ has zero-dimensional stabiliser in $\mathscr{G}_{F \oplus F^{\perp}}^{0}$ if and only if $\left(\Psi, A_{1} \oplus A_{2}\right)$ does so in $\mathscr{G}^{0}$. Now we can state the following:

Proposition 2.10. Restriction of the map $i_{F}$ yields an injective map

$$
i_{F}: \mathscr{B}_{\mathfrak{s}, E, \theta}^{*, i r r} F \oplus F^{\perp} \rightarrow \mathscr{B}_{\mathfrak{s}, E, \theta}^{*[F]}
$$

from the subset $\mathscr{B}_{\mathfrak{s}, E, \theta}^{*, i r r} F \oplus F^{\perp}$ of configurations, up to gauge, with zero-dimensional stabilisers inside $\mathscr{G}_{F \oplus F^{\perp}}^{0}$ and irreducible connections, into the fixed-point set component $\mathscr{B}_{\mathfrak{s}, E, \theta}^{*[F]}$ of configurations, up to gauge, with zero-dimensional stabilisers inside $\mathscr{G}^{0}$.

Proof: For simplicity we note $i$ instead of $i_{F}$. Suppose we have elements [ $\left.\Psi, A_{1}, A_{2}\right]$, $\left[\Phi, B_{1}, B_{2}\right] \in \mathscr{B}_{\mathfrak{s}, E, \theta}^{*, i r r} F \oplus F^{\perp}$ such that $i\left(\left[\Psi, A_{1}, A_{2}\right]=i\left[\Phi, B_{1}, B_{2}\right]\right.$. This is equivalent to saying that there is a gauge transformation $u \in \mathscr{G}^{0}$ such that

$$
\begin{aligned}
u(\Psi) & =\Phi \\
u\left(A_{1} \oplus A_{2}\right) & =B_{1} \oplus B_{2} .
\end{aligned}
$$

The second equation implies that $u$ is an $\left(A_{1} \oplus A_{2}\right) \otimes\left(B_{1} \oplus B_{2}\right)^{*}$-parallel endomorphism of $E$. Let us write $u$ in the form

$$
\left(\begin{array}{ll}
u_{11} & u_{12} \\
u_{21} & u_{22}
\end{array}\right)
$$

according to the splitting $E=F \oplus F^{\perp}$. Injectivity will follow if we have $u_{12}=0$ and $u_{21}=0$. It is enough to show just $u_{21}=0$, as the other equation will follow from the fact that $u$ is unitary. We find that the morphism $u_{11}$ is $B_{1} \otimes A_{1}^{*}$-parallel, the morphism $u_{12}$ is $B_{1} \otimes A_{2}^{*}$-parallel, the morphism $u_{21}$ is $B_{2} \otimes A_{1}^{*}$-parallel, and the morphism $u_{22}$ is $B_{2} \otimes A_{2}^{*}$-parallel .

Now all the connections $A_{i}, B_{i}, i=1,2$ are unitary connections. Therefore the fact that, for instance, $u_{12}$ is $B_{1} \otimes A_{2}^{*}$-parallel implies that the adjoint $u_{12}^{*}$ is $A_{2} \otimes B_{1}^{*}$ parallel. As a consequence, the endomorphism $u_{21}^{*} u_{21}$ of $F$ is $A_{1} \otimes A_{1}^{*}$-parallel, and the endomorphism $u_{12}^{*} u_{12}$ of $F^{\perp}$ is $A_{2} \otimes A_{2}^{*}$-parallel. By the hypothesis $A_{1}$ and $A_{2}$ are irreducible, so that the above Lemma 2.2 implies that there are constants $\xi, \zeta \in \mathbb{C}$ with

$$
\begin{aligned}
& u_{21}^{*} u_{21}=\xi \operatorname{id}_{F} \\
& u_{12}^{*} u_{12}=\zeta \operatorname{id}_{F^{\perp}} .
\end{aligned}
$$

We have to show now that under our hypothesis $\xi=0$ or $\zeta=0$, implying then that $u_{21}=0$ respectively $u_{12}=0$. But if we had $\xi \neq 0$, then $u_{21}$ is injective at each point $x \in X$. By the hypothesis we get that $\Psi \neq 0$, and therefore we would have a non-trivial section $u_{21}(\Psi) \in S_{\mathfrak{s}}^{+} \otimes F^{\perp}$. However, we have $u(\Psi)=\Phi$, where $\Phi$ is a section of $S_{\mathfrak{s}}^{+} \otimes F$, so that this would yield a contradiction. Therefore $\xi=0$ and as a consequence $u_{21}=0$ and $u_{12}=0$. 
Next we shall discuss a canonical fibering of the configuration space up to gauge respecting the proper decomposition $E=F \oplus F^{\perp}$ that we have introduced above. Let us denote now by $\mathscr{G}_{F}^{0}$ the group of special unitary automorphisms of the unitary bundle $F$ on $X$, that is $\mathscr{G}_{F}^{0}=\Gamma(X, S U(F))$. So, with this notation, $\mathscr{G}_{E}^{0}$ is the gauge group we have until now denoted by the letter $\mathscr{G}^{0}$. On the other hand, we shall denote by $\mathscr{G}_{F}$ the group of unitary automorphisms of $F$, that is, $\mathscr{G}_{F}=\Gamma(X, U(F))$.

Lemma 2.11. We have an exact sequence of groups given by

$$
1 \rightarrow \mathscr{G}_{F \perp}^{0} \stackrel{i}{\rightarrow} \mathscr{G}_{F \oplus F^{\perp}}^{0} \stackrel{j}{\rightarrow} \mathscr{G}_{F} \rightarrow 1 .
$$

Here the morphisms are given by $i\left(u_{2}\right)=\left(i d_{F}, u_{2}\right)$ and $j\left(\left(u_{1}, u_{2}\right)\right):=u_{1}$.

Proof: The only non-trivial point is the surjectivity of the morphism $j$. Indeed, for a given gauge transformation $u_{2} \in \mathscr{G}_{F}$ we have to find some automorphism $u_{1} \in \mathscr{G}_{F^{\perp}}$ such that $\operatorname{det}\left(u_{1}\right) \cdot \operatorname{det}\left(u_{2}\right)=1$. So we have to find an automorphism of $F^{\perp}$ with prescribed determinant $\operatorname{det}\left(u_{2}\right)^{-1}$. That this is indeed possible follows from obstruction theory [25] [18].

We shall introduce some new notation now. Given a Hermitian vector bundle $F$ on $X$ we shall denote by $P_{F}$ its associated frame bundle, a principal bundle of structure group $U(n)$, where $n$ is the rank of $F$. Let us denote by $\mathscr{A}_{P U}(F)$ the affine space of connections in the associated $P U(n)$-bundle $P_{F} \times_{\pi} P U(n)$, where $\pi$ is the natural projection $U(n) \rightarrow P U(n)$. Note that in the case that $n=\operatorname{rank}(F)=1$ the bundle $P_{F} \times_{\pi} P U(n)$ is the trivial principal bundle with structure group the trivial group, and both $\mathscr{A}_{P U}(F)$ and $\mathscr{A}_{P U}(F) / \mathscr{G}_{F}^{0}$ consist of a single point.

Definition 2.12. We shall denote by $\mathscr{B}_{F}^{P U}$ the set of all $P U(n)$-connections $A \in$ $\mathscr{A}_{F}^{P U}$ in the unitary bundle $F$ up to the gauge group $\mathscr{G}_{F}^{0}$ of special unitary automorphisms of the bundle $F$. Notice that up to specifying a connection $\vartheta \in \operatorname{det}(F)$ we have isomorphisms $\mathscr{A}_{F}^{P U} \equiv \mathscr{A}_{\vartheta}(F)$ and likewise $\mathscr{B}_{F}^{P U} \equiv \mathscr{B}_{\vartheta}(F)$, with the notations of Section 1.

Proposition 2.13. Suppose the 4-manifold $X$ is simply connected. Then we have a bijection

$$
\begin{aligned}
h: \mathscr{B}_{\mathfrak{s}, E, \theta}^{F \oplus F^{\perp}} \stackrel{\cong}{\rightrightarrows} \mathscr{B}_{\mathfrak{s}, F} \times \mathscr{B}_{F^{\perp}}^{P U} \\
{\left[\Psi, A_{1}, A_{2}\right] \mapsto\left(\left[\Psi, A_{1}\right],\left[A_{2}\right]\right) . }
\end{aligned}
$$

Proof: We leave the proof of this proposition to the reader and only notice that the simply-connectedness of $X$ is used to ensure the existence of roots of $U(1)$ valued functions on $X$. In fact some (non-canonical) $(N-n)$ th root of $\operatorname{det}\left(u_{1}\right)$ will appear. For a complete proof see [31].

Remark. Without the assumption that $X$ is simply-connected we can still show that we get a fibration $\mathscr{B}_{\mathfrak{s}, E}^{F \oplus F^{\perp}} \rightarrow \mathscr{B}_{\mathfrak{s}, F}^{U}$ with standard fibre $\mathscr{A}_{P U}\left(F^{\perp}\right) / \mathscr{G}_{F}^{0}$. The nontriviality of this fibration should be encoded in $H_{1}(X, \mathbb{Z})$. From now on, however, we shall suppose that our 4-manifold $X$ is simply connected. 
2.5. The circle-action on the moduli space of $P U(N)$-monopoles. Until now our consideration of the $S^{1}$-action and its fixed point set was inside the configuration space up to gauge, $\mathscr{B}_{\mathfrak{s}, E, \theta}$. Obviously, the moduli space of $P U(N)$-monopoles $M_{\mathfrak{s}, E, \theta} \subseteq \mathscr{B}_{\mathfrak{s}, E, \theta}$ is invariant under the $r$-action, $r\left(S^{1}, M_{\mathfrak{s}, E}\right) \subseteq M_{\mathfrak{s}, E}$. All we have found out about the circle-action on $\mathscr{B}_{\mathfrak{s}, E, \theta}$ applies to the restriction of this action to the moduli space as well. However, there are more things we can say about the fixed-point set of the circle-action for this restriction. In particular, these fixed-point sets are naturally related to other moduli spaces. Obviously the

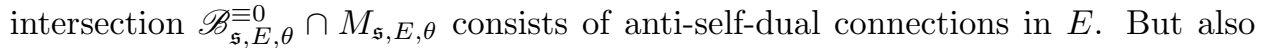
the intersection $\mathscr{B}_{\mathfrak{s}, E, \theta}^{[F]} \cap M_{\mathfrak{s}, E, \theta}$ is parametrised by the product of the moduli space of $U(n)$-monopoles in $F$, with $n=\operatorname{rk}(F)$, and the moduli space of anti-self-dual $P U(N-n)$-connections in $F^{\perp}$, as we shall see.

Proposition 2.6 above described the fixed-points of the circle-action on $\mathscr{B}_{\mathfrak{s}, E, \theta}$, the configuration space modulo gauge. In particular the element $[\Psi, A]$ lies in $\mathscr{B}_{\mathfrak{s}, E, \theta}^{[F]}$ if and only if for a representative $(\Psi, A)$ we have a (proper) $A$-parallel decomposition $E=F \oplus F^{\perp}$, and the spinor part $\Psi$ is a section of $S_{\mathfrak{s}}^{+} \otimes F$. This second condition becomes automatically satisfied if $(\Psi, A)$ solve the $P U(N)$ monopole equations:

Proposition 2.14. Suppose the configuration $(\Psi, A)$ satisfies the PU(N)-SeibergWitten-equations (4) associated to the data $(\mathfrak{s}, E)$.

Suppose further that the connection $A$ is reducible, and that $E=\oplus E_{i}$ is a A-parallel orthogonal decomposition into proper subbundles, and that the base manifold $X$ is connected. Then the spinor must be a section of one of the bundles $S_{\mathfrak{s}}^{+} \otimes E_{i}$.

Proof: Suppose the connection $A$ splits into two connections $A_{1} \oplus A_{2}$ with respect to $E=E_{1} \oplus E_{2}$. As an endomorphism of $E$ the curvature $F_{A}$ splits as

$$
F_{A}=\left(\begin{array}{cc}
F_{A_{1}} & 0 \\
0 & F_{A_{2}}
\end{array}\right) \text {. }
$$

In other words, it is a section of $\Lambda^{2}\left(T^{*} X\right) \otimes\left(\mathfrak{u}\left(E_{1}\right) \oplus \mathfrak{u}\left(E_{2}\right)\right)$. The trace-free part $F_{A}$ is then a section of the bundle $\Lambda^{2}\left(T^{*} X\right) \otimes\left(\left(\mathfrak{u}\left(E_{1}\right) \oplus \mathfrak{u}\left(E_{2}\right)\right) \cap \mathfrak{s u}(E)\right)$. Therefore the curvature-equation of the $P U(N)$-monopole-equations implies that

$$
\mu_{0,0}(\Psi) \in \Gamma\left(X, \mathfrak{s u}\left(S_{\mathfrak{s}}^{+}\right) \otimes\left(\left(\mathfrak{u}\left(E_{1}\right) \oplus \mathfrak{u}\left(E_{2}\right)\right) \cap \mathfrak{s u}(E)\right)\right) .
$$

Now decompose the spinor as $\Psi=\Psi_{1}+\Psi_{2}$, where $\Psi_{i} \in \Gamma\left(X, S_{\mathfrak{s}}^{+} \otimes E_{i}\right)$. Recall that the quadratic map $\mu_{0,0}$ is defined to be $\mu_{0,0}(\Psi)=\mu_{0,0}(\Psi, \Psi)$, where on the right we mean the bilinear map $\mu_{0,0}$. We get

$$
\mu_{0,0}(\Psi, \Psi)=\mu_{0,0}\left(\Psi_{1}, \Psi_{1}\right)+\mu_{0,0}\left(\Psi_{1}, \Psi_{2}\right)+\mu_{0,0}\left(\Psi_{2}, \Psi_{1}\right)+\mu_{0,0}\left(\Psi_{2}, \Psi_{2}\right) .
$$

By the definition of $\mu_{0,0}$ and by the above equation (10) we see that $\mu_{0,0}\left(\Psi_{1}, \Psi_{2}\right)=$ $\mu_{0,0}\left(\Psi_{2}, \Psi_{1}\right)=0$. Now from the fact that the bilinear map $\mu_{0,0}$ is "without zerodivisors' by the above Proposition 1.1 we see that in each fibre $\Psi_{1}=0$ or $\Psi_{2}=0$. Suppose we have $\Psi_{1}\left(x_{0}\right) \neq 0$ for some point $x_{0} \in X$. As $\Psi$ is continuous we must have $\Psi \neq 0$ for all $x$ in some neighbourhood $U$ of $x_{0}$. Therefore $\Psi_{2} \equiv 0$ on $U$. However, the Dirac equation $\varnothing_{A}^{+} \Psi=0$ implies that $\not_{A_{1}}^{+} \Psi_{1}=0$ and that $\not_{A_{2}}^{+} \Psi_{2}=0$, where the Dirac operator $D_{A_{i}}^{+}: \Gamma\left(X, S_{\mathfrak{s}}^{+} \otimes E_{i}\right) \rightarrow \Gamma\left(X, S_{\mathfrak{s}}^{-} \otimes E_{i}\right)$ is defined to be the composition of $\nabla_{A_{i}, B}: \Gamma\left(X, S_{\mathfrak{s}}^{+} \otimes E_{i}\right) \rightarrow \Omega^{1}\left(X, S_{\mathfrak{s}}^{+} \otimes E_{i}\right)$ with the Clifford-map $\gamma: T^{*} X \otimes\left(S_{\mathfrak{s}}^{+} \otimes E_{i}\right) \rightarrow\left(S_{\mathfrak{s}}^{-} \otimes E_{i}\right)$. But for each of these Dirac operators there is a unique continuation theorem for elements in its kernel by Aronaszajin's Theorem 
1]. Therefore, as $\Psi_{2} \equiv 0$ on $U$, it must vanish identically on $X$. The general case follows easily by iterating the same argument.

Remark. The $S^{1}$-action extends naturally to the Uhlenbeck-compactification

$$
\bar{M}_{\mathfrak{s}, E, \theta} \subset I M_{\mathfrak{s}, E, \theta}=\coprod_{k \geq 0} M_{\mathfrak{s}, E_{-k}, \theta} \times \operatorname{Sym}^{k}(X)
$$

(with its above-defined topology).

Another important result is the following finiteness property of the fixed-point locus inside the (compactified) moduli space:

Proposition 2.15. Given the data $(\mathfrak{s}, E)$ the respective moduli spaces $M_{\mathfrak{s}, E_{-k}, \theta}$, $k \geq 0$, occuring in the definition of the Uhlenbeck compactification of $M_{\mathfrak{s}, E, \theta}$, intersect the respective fixed point loci $\mathscr{B}_{\mathfrak{s}, E_{-k}, \theta}^{[F]}$ only in finitely many isomorphism classes of proper summands $[F]$ of $E$.

Proof: We will show that if $[\Psi, A] \in M_{\mathfrak{s}, E} \cap \mathscr{B}_{\mathfrak{s}, E}^{[F]}$, then $c_{1}^{\mathbb{R}}(F)$ lies in a bounded set within $H^{2}(X, \mathbb{R})$, and $\left\langle c_{2}(F),[X]\right\rangle \in \mathbb{Z}$ is bounded also. As $c_{1}^{\mathbb{R}}(F)$ is in the image of the morphism $H^{2}(X, \mathbb{Z}) \rightarrow H^{2}(X, \mathbb{R})$, it will follow that $c_{1}(F)$ lies in a finite set. The conclusion is then that only finitely many pairs $\left(c_{1}, c_{2}\right) \in H^{2}(X, \mathbb{Z}) \times H^{4}(X, \mathbb{Z})$ can occur as first and second Chern-class of $F$. But on a closed oriented 4-manifold unitary bundles are classified, up to isomorphism, by their first and second Chern class.

Recall the Chern-Weil formulae for the image of the first and second Chern class inside $H^{*}(X, \mathbb{R}) \cong H_{d R}^{*}(X)$ :

$$
\begin{aligned}
c_{1}^{\mathbb{R}}(E) & =\frac{-1}{2 \pi i}\left[\operatorname{tr} F_{A}\right], \\
c_{2}^{\mathbb{R}}(E) & =\frac{-1}{4 \pi^{2}}\left[\frac{1}{2}\left(\operatorname{tr} F_{A} \wedge \operatorname{tr} F_{A}-\operatorname{tr}\left(F_{A} \wedge F_{A}\right)\right)\right] \\
& =\frac{1}{2}\left\langle c_{1}(E)^{2},[X]\right\rangle+\frac{1}{8 \pi^{2}}\left(\left\|F_{A}^{-}\right\|_{L^{2}(X)}^{2}-\left\|F_{A}^{+}\right\|_{L^{2}(X)}^{2}\right) .
\end{aligned}
$$

The vector space $H^{2}(X ; \mathbb{R})$ is isomorphic to the space of harmonic 2-forms $\mathscr{H}^{2}(X, g)$. For each class $[\omega] \in H^{2}(X ; \mathbb{R})$ its harmonic representative $\omega_{g}$ is minimising the $L^{2}$ norm among all representatives of the same class. So if we give the space $H^{2}(X ; \mathbb{R})$ the inner product via its identification with $\mathscr{H}^{2}(X, g)$ we see that a subset of $H^{2}(X ; \mathbb{R})$ is bounded if the $L^{2}$ norms of a set of representative forms is bounded. Thus to bound the classes $c_{1}(F)=\left[\operatorname{tr} F_{A_{1}}\right]$ it is enough to bound the norms

$$
\left\|\operatorname{tr} F_{A_{1}}\right\|_{L^{2}(X)}
$$

with $A_{1}$ a connection on the summand $F$ of $E$.

Now by the assumption that $[\Psi, A] \in M_{\mathfrak{s}, E} \cap \mathscr{B}_{\mathfrak{s}, E}^{[F]}$ we have a connection $A$ on $E$ that reduces to $A_{1} \oplus A_{2}$ according to the splitting $E=F \oplus F^{\perp}$. We therefore get the decomposition

$$
F_{A}=\left(\begin{array}{cc}
F_{A_{1}} & 0 \\
0 & F_{A_{2}}
\end{array}\right) \text {. }
$$


In particular, we have bounds

$$
\begin{aligned}
\left\|\operatorname{tr} F_{A_{1}}\right\|_{L^{2}(X)} & \leq\left\|\operatorname{tr} F_{A}\right\|_{L^{2}(X)} \\
\left\|F_{A_{1}}^{+}\right\|_{L^{2}(X)} & \leq\left\|F_{A}^{+}\right\|_{L^{2}(X)} \\
\left\|F_{A_{1}}^{-}\right\|_{L^{2}(X)} & \leq\left\|F_{A}^{-}\right\|_{L^{2}(X)} .
\end{aligned}
$$

However, by the $P U(N)$ monopole equations (4), the a-prioi bound (6), and the Chern-Weil formula for $c_{2}$ we see that the quantities on the right hand sides are bounded given by expressions that depend on the metric $g$, the $\operatorname{Spin}^{c}$ connection $B$ and the connection $\theta$ in the determinant line bundle, as well as on some constants related to the $\mu$-map and $\gamma$, so these are uniformly bounded on $M_{\mathfrak{s}, E, \theta}$.

By the Chern-Weil formulae we therefore see that $c_{1}(F)$ is uniformly bounded in $H^{2}(X, \mathbb{R})$, and therefore $\left|\left\langle c_{2}(F),[X]\right\rangle\right|$ is likewise bounded. As the apriori-bound on the spinor (6) does not depend on the second Chern class of $E$ it follows that there is a corresponding statement for the lower strata of the Uhlenbeck compactification.

In the sequal we shall denote by $M_{\mathfrak{s}, E}^{S^{1}}$ the intersection $\mathscr{B}_{\mathfrak{s}, E}^{S^{1}} \cap M_{\mathfrak{s}, E}$, as well as by $M_{\mathfrak{s}, E}^{[F]}$ the intersection of $\mathscr{B}_{\mathfrak{s}, E}^{[F]} \cap M_{\mathfrak{s}, E}$. Also, $M_{\mathfrak{s}, E}^{*}$ shall denote the intersection of $\mathscr{B}_{\mathfrak{s}, E}^{*}$ with $M_{\mathfrak{s}, E}$, and $M_{\mathfrak{s}, E}^{* S^{1}}, M_{\mathfrak{s}, E}^{*}[F]$ the respective intersections with the fixed point set and the given fixed point set component.

2.6. Monopole equations for configurations mapping to the fixed point set. Above we have pointed out that for describing the component of the fixed point set $\mathscr{B}_{\mathfrak{s}, E}^{[F]}$ determined by the isomorphism class of a proper subbundle $[F]$ of $E$, it is useful to keep a representative $F$ fixed. We did then describe the component $\mathscr{B}_{\mathfrak{s}, E}^{[F]}$ as the image via $i_{F}$ of the space $\mathscr{B}_{\mathfrak{s}, E}^{F \oplus F^{\perp}}$ which is easier to handle with. It will turn out that this way we also get a convenient description of $M_{\mathfrak{s}, E}^{[F]}$, which we define to be the intersection of $\mathscr{B}_{\mathfrak{s}, E}^{[F]}$ with the moduli space $M_{\mathfrak{s}, E}$.

Let us write down explicitly the monopole equations which are satisfied by a representative $(\Psi, A)$ having the property that there is a $A$-parallel decomposition of $E$ into $F \oplus F^{\perp}$, with $\Psi$ a section of $S_{\mathfrak{s}}^{+} \otimes F$, and $A$ splitting as $A_{1} \oplus A_{2}$. Recall that $\operatorname{det}(A)=\operatorname{det}\left(A_{1}\right) \otimes \operatorname{det}\left(A_{2}\right)$ is the fixed connection $\theta$ in the determinant line bundle $\operatorname{det}(E)$. We then have

$$
\left(F_{A}\right)_{0}=F_{A}-\frac{1}{N} \operatorname{tr}\left(F_{A}\right) \mathrm{id}_{E}=\left(\begin{array}{cc}
F_{A_{1}}-\frac{1}{N} F_{\theta} & 0 \\
0 & F_{A_{2}}-\frac{1}{N} F_{\theta}
\end{array}\right),
$$

according to the splitting $E=F \oplus F^{\perp}$, and also

$$
\begin{aligned}
\mu_{0,0}(\Psi) & =\left(\begin{array}{ccc}
\mu_{0,1}^{F}(\Psi)-\frac{1}{N} \operatorname{tr} \mu_{0,1}^{F}(\Psi) \operatorname{id} & 0 \\
0 & -\frac{1}{N} \operatorname{tr} \mu_{0,1}^{F}(\Psi) \operatorname{id}_{F^{\perp}}
\end{array}\right) \\
& =\left(\begin{array}{ccc}
\mu_{0,1-\frac{n}{N}}^{F}(\Psi) & 0 \\
0 & -\frac{1}{N} \operatorname{tr} \mu_{0,1}^{F}(\Psi) \operatorname{id}_{F^{\perp}}
\end{array}\right) .
\end{aligned}
$$


The $P U(N)$-monopole equations (4) for the pair $\left(\Psi, A_{1} \oplus A_{2}\right)$ then read

$$
\begin{aligned}
\not_{A_{1}}^{+} \Psi & =0 \\
\gamma\left(F_{A_{1}}^{+}\right)-\mu_{0,1-\frac{n}{N}}^{F}(\Psi) & =\frac{1}{N} \gamma\left(F_{\theta}^{+}\right) \mathrm{id}_{F} \\
\gamma\left(F_{A_{2}}^{+}\right)+\frac{1}{N} \operatorname{tr} \mu_{0,1}^{F}(\Psi) \mathrm{id}_{F^{\perp}} & =\frac{1}{N} \gamma\left(F_{\theta}^{+}\right) \mathrm{id}_{F^{\perp}} .
\end{aligned}
$$

Here the terms in the second equation are sections of the bundle $\mathfrak{s u}\left(S_{\mathfrak{s}}^{+}\right) \otimes_{\mathbb{R}} \mathfrak{u}(F)$ and the terms in the third equation are sections of $\mathfrak{s u}\left(S_{\mathfrak{s}}^{+}\right) \otimes_{\mathbb{R}} \mathfrak{u}\left(F^{\perp}\right)$. There is Lie algebra decompositions $\mathfrak{u}(F)=\mathfrak{s u}(F) \oplus i \mathbb{R}$ and correspondingly for $\mathfrak{u}\left(F^{\perp}\right)$. It turns

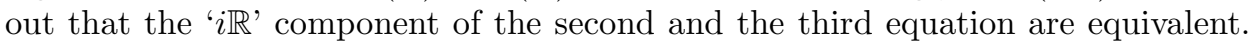
Indeed, taking the trace (with respect to the factor $\mathfrak{u}(F)$ in $\mathfrak{s u}\left(S_{\mathfrak{s}}^{+}\right) \otimes_{\mathbb{R}} \mathfrak{u}(F)$, and correspondingly for $\mathfrak{u}\left(F^{\perp}\right)$ ) of the second and the third equation, and using the fact that

$$
\operatorname{tr}\left(F_{A_{1}}\right)+\operatorname{tr}\left(F_{A_{2}}\right)=F_{\theta}
$$

this follows from a simple computation. Therefore the system of equations (12) above is equivalent to the same system where we take as the third equation only the component of $\mathfrak{s u}\left(F^{\perp}\right)$ according to $\mathfrak{u}\left(F^{\perp}\right)=\mathfrak{s u}\left(F^{\perp}\right) \oplus i \mathbb{R}$. Thus the $P U(N)$ monopole equations are therefore equivalent to

$$
\begin{aligned}
D_{A_{1}}^{+} \Psi & =0 \\
\gamma\left(F_{A_{1}}^{+}\right)-\mu_{0,1-\frac{n}{N}}^{F}(\Psi) & =\frac{1}{N} \gamma\left(F_{\theta}^{+} \mathrm{id}_{F}\right) \\
\left(F_{A_{2}}^{+}\right)_{0} & =0 .
\end{aligned}
$$

The first two equations of (13) are $U(n)$ - monopole equations for $\left(\Psi, A_{1}\right)$ with parameters $\tau=1-\frac{n}{N} \in[0,1]$ and self-dual 2-form $\eta=\frac{1}{N} F_{\theta}^{+}$, and the third equation is the anit-self-duality equation for the $P U(N-n)$ connection $A_{2}$. We shall denote by $M_{F}^{\text {asd }}$ the moduli space of anti-self-dual $P U(n)$ - connections in $F$ which is defined to be the space of $P U(n)$ - connections $A \in \mathscr{A}^{P U}(F)$ in $F$ which satisfy the equations $F_{A}^{+}=0$, quotiented by the action of the gauge-group $\mathscr{G}_{F}^{0}$ of special unitary automorphisms of $F$. Equivalently, if we think of $A$ as a unitary connection under an isomorphism $\mathscr{A}^{P U}(F) \cong \mathscr{A}_{\vartheta}(F)$ specified by a fixed connection $\vartheta$ in the determinant line bundle $\operatorname{det}(F)$, the (projective) anti-selfduality equation for $A$ becomes $\left(F_{A}^{+}\right)_{0}=0$.

We summarise this computation in the following:

Proposition 2.16. Suppose the configuration $(\Psi, A) \in \mathscr{C}_{\mathfrak{s}, E}$ has reducible connection part $A=A_{1} \oplus A_{2}$ according to $E=F \oplus F^{\perp}$, and that the spinor part $\Psi$ is a section of $S^{+} \otimes F$ (compare proposition 2.14). Then the $P U(N)$ monopole equations for $(\Psi, A)$ are equivalent to the system (13)). In particular, the configuration $\left(\Psi, A_{1}\right)$ represents a $U(n)$ monopole in the moduli space $M_{\mathfrak{s}, F}\left(1-\frac{n}{N}, \frac{1}{N} F_{\theta}^{+}\right)$, and the connection $A_{2}$ represents an instanton in the moduli space $M_{F^{\perp}}^{a s d}$.

Definition 2.17. We shall denote by $M_{\mathfrak{s}, E}^{F \oplus F^{\perp}} \subseteq \mathscr{B}_{\mathfrak{s}, E}^{F} F^{\perp}$ the moduli space space of solutions $\left(\Psi, A_{1}, A_{2}\right)$ to the above equations (13) modulo the gauge group $\mathscr{G}_{F \oplus F^{\perp}}^{0}$. As usually, we denote by $M_{\mathfrak{s}, E}^{* F} F^{\perp}$ the subspace of those elements whose representatives have zero-dimensional stabiliser. 
Proposition 2.18. The map $i_{F}: \mathscr{B}_{\mathfrak{s}, E}^{F \oplus F^{\perp}} \rightarrow \mathscr{B}_{\mathfrak{s}, E}^{[F]}$ maps the moduli space $M_{\mathfrak{s}, E}^{F \oplus F^{\perp}}$ onto the fixed point set component $M_{\mathfrak{s}, E}^{[F]}$ inside the moduli space. It maps the set $M_{\mathfrak{s}, E}^{*} F \oplus F^{\perp}$ bijectively onto $M_{\mathfrak{s}, E}^{*[F]}$.

Proof: The fact that the map is onto is an immediate consequence of the above Proposition 2.16 and the definition of $M_{\mathfrak{s}, E}^{[F]}$. For the remaining claim we will show that we can apply the above Proposition 2.10. First, we shall observe that if $\left[\Psi, A_{1}, A_{2}\right]$ belongs to $M_{\mathfrak{s}, E}^{* F \oplus F^{\perp}}$, then the connections $A_{1}$ and $A_{2}$ are indeed irreducible. Obviously $A_{2}$ has to be irreducible, but suppose $A_{1}$ were reducible. We would have a $A_{1}$ - parallel orthogonal decomposition $F=F_{1} \oplus F_{2}$, with $A_{1}$ splitting accordingly, $A_{1}=A_{11} \oplus A_{12}$.

Let us write $\Psi=\Psi_{1}+\Psi_{2} \in \Gamma\left(X, S_{\mathfrak{s}}^{+} \otimes\left(F_{1} \oplus F_{2}\right)\right)$ for the corresponding decomposition of the spinor. We claim that either $\Psi_{1}=0$ or $\Psi_{2}=0$. In fact, $\left(\Psi, A_{1}\right)$ solves the first two of the equations (13). The map $\mu_{0, \tau}^{F}$ is 'without zero-divisors' by the above Proposition 1.1. With this fact the conclusion follows exactly like in the proof of Proposition 2.14 But then the configuration $\left(\Psi, A_{11} \oplus A_{12}, A_{2}\right)$ must have positive-dimensional stabiliser inside $\mathscr{G}_{F \oplus F^{\perp}}^{0}$, and the element $\left[\Psi, A_{1}, A_{2}\right]$ would not belong to $M_{\mathfrak{s}, E}^{* F \oplus F^{\perp}}$. Therefore $\left[\Psi, A_{1}, A_{2}\right]$ belongs to the set $\mathscr{B}_{\mathfrak{s}, E}^{*, i r r} F \oplus F^{\perp}$ and we can apply Proposition 2.10 for getting injectivity. Furthermore it is easy to see that the parametrisation $i_{F}$ maps $M_{\mathfrak{s}, E}^{*} F \oplus F^{\perp}$ onto $M_{\mathfrak{s}, E}^{*[F]}$.

Proposition 2.19. Restricting the bijection $h$ of Proposition 2.13 above to the moduli space $M_{\mathfrak{s}, E, \theta}^{F \oplus F^{\perp}}$ we get an induced bijection

$$
\left.h\right|_{M}: M_{\mathfrak{s}, E, \theta}^{F \oplus F^{\perp}} \stackrel{\cong}{\rightrightarrows} M_{\mathfrak{s}, F}\left(1-n / N, 1 / N F_{\theta}^{+}\right) \times M_{F^{\perp}}^{a s d}
$$

Together with the map $i_{F}$ we thus get the parametrisation of the fixed point set component $M_{\mathfrak{s}, E}^{[F]}$ of the moduli space as the product of a moduli space of $U(n)$ monopoles with the moduli space of $A S D-P U(N-n)$-connections. In particular, for the irreducible parts we get a bijection

$$
\left.i_{F} \circ h\right|_{M} ^{-1}: M_{\mathfrak{s}, F}^{*}\left(1-n / N, 1 / N F_{\theta}^{+}\right) \times M_{F^{\perp}}^{* \text { asd }} \cong M_{\mathfrak{s}, E, \theta}^{*[F]} .
$$

This follows from Proposition 2.18 and Proposition 2.13, where it is easily checked that $\left.h\right|_{M} ^{-1}$ maps the 'irreducibles' $M_{\mathfrak{s}, F}^{*} \times M_{F^{\perp}}^{* \text { asd }}$ onto the corresponding 'irreducibles' $M_{\mathfrak{s}, E, \theta}^{* F \oplus F^{\perp}}$.

The whole discussion is now summarised in

Theorem 2.20. The fixed point set under the above circle-action $r$ on the moduli space $M_{\mathfrak{s}, E, \theta}$ of $P U(N)$ monopoles is given as the union of the moduli space $M_{E}^{\text {asd }}$ of anti-self-dual $P U(N)$ connections in $E$ and a finite union

$$
\bigcup_{[F] \subseteq E} M_{\mathfrak{s}, E}^{[F]}
$$

of components $M_{\mathfrak{s}, E}^{[F]}$ indexed by a finite number of isomorphism classes [F] of proper subbundles of $E$. The spaces $M_{\mathfrak{s}, E}^{[F]}$ are given as follows: An element $[\Psi, A]$ belongs 
to $M_{\mathfrak{s}, E}^{[F]}$ if for each representative $F \in[F]$ there is a representative $(\Psi, A) \in[\Psi, A]$ such that $F$ is an A-invariant proper subbundle of $E$ and the spinor $\Psi$ is a section of the proper subbundle $S_{\mathfrak{s}}^{+} \otimes F$ of $W_{\mathfrak{s}, E}^{+}=S_{\mathfrak{s}}^{+} \otimes E$.

Furthermore, if $X$ is simply connected, then there is a parametrisation of this space $M_{\mathfrak{s}, E, \theta}^{[F]}$ as the product

$$
M_{\mathfrak{s}, F}\left(1-n / N, 1 / N F_{\theta}^{+}\right) \times M_{F^{\perp}}^{a s d} \rightarrow M_{\mathfrak{s}, E, \theta}^{[F]} .
$$

This map is a surjection and is a bijection between the open and dense subsets of elements with zero-dimensional stabiliser in the corresponding moduli spaces,

$$
M_{\mathfrak{s}, F}^{*}\left(1-n / N, 1 / N F_{\theta}^{+}\right) \times M_{F^{\perp}}^{*} \stackrel{\text { asd }}{\cong} M_{\mathfrak{s}, E, \theta}^{*[F]} .
$$

We observe that there is a corresponding statement if we take the whole Uhlenbeckcompactification of $M_{\mathfrak{s}, E, \theta}$ into account.

\section{What TO EXPECT FROM THE COBORDISM PROGRAM}

The heuristical idea of the cobordism program is that the $S^{1}$-quotient of the complement of the fixed point locus $M_{\mathfrak{s}, E, \theta} \backslash M_{\mathfrak{s}, E, \theta}^{S^{1}}$ yields an oriented cobordism between

(1) a projective bundle over the instanton moduli space $M_{E}^{a s d}$, the fibre over an instanton $A$ being the projectivisation of the kernel of the dirac operator $\not D_{A}^{+}$, and

(2) projective bundles over the moduli spaces $M_{\mathfrak{s}, F} \times M_{F^{\perp}}^{\text {asd }}$, corresponding to a parallel decompositions $E=F \oplus F^{\perp}$, the fibres being projectivisation of the (complex) normal bundles that can be described by local models around these $S^{1}$ fixed point spaces.

Then there are extension of the Donaldson- $\mu$-classes (see [5, Section 5 and 9] for instance), lifted to the projective bundle occuring in (1) (and multiplied with a power of the first Chern class of the bundle of kernels of the Dirac operator), to the whole $S^{1}$-quotient. Therefore, the higher rank instanton invariants, obtained by evaluating the $\mu$-classes on the 'fundamental cylce' given by the moduli space $M_{E}^{a s d}$, should be expressible by corresponding evaluations involving the spaces $M_{\mathfrak{s}, F} \times$ $M_{\mathfrak{s}, F^{\perp}}^{a s d}$.

The components of the second type involving $M_{\mathfrak{s}, F}$ for $r k(F)=1$ are contributions to this evaluation that are expected to involve the Seiberg-Witten invariants of the $\operatorname{Spin}^{c}$ structure $\mathfrak{s} \otimes F$. The corresponding generalisation of Witten's conjecture would follow then by induction on the rank $N$ if the the moduli spaces $M_{\mathfrak{s}, F}$ for $r k(F)>1$ contributed trivially to a cobordism-formula for the higher rank invariant to which we made allusion. We present two arguments why such contributions can be expected to vanish. The second, by a consideration on Kähler surfaces, is obtained in the next section. The first, admittedly less convincing, shall be outlined in the remainder of this section.

First, let us recall that the $U(n)$ monopole moduli spaces $M_{\mathfrak{s}, F}(\tau)$ admit an Uhlenbeck compactification for any $\tau \in[0,1]$ for we still obtain an apriori-bound if 
$n>1$. Let us assume we have a space of perturbations $\mathscr{P}$ such that the irredubible part of the 'parametrised moduli space', the zero-locus of the resulting map

$$
\mathscr{B}_{\mathfrak{s}, F}^{* *} \times \mathscr{P} \times[0,1] \rightarrow \Gamma\left(X ; S_{\mathfrak{s}}^{-} \otimes F \oplus \Lambda_{+}^{2}(\mathfrak{u}(F)),\right.
$$

with $[0,1]$ being the parameter space for $\tau$, is cut out transversally. The existence of such a parameter space, compatible with some kind of Uhlenbeck compactification for the moduli space, is not speculative, see for instance 9 for the case $n=2$ or 32 for general $n$ (but less general with respect to Uhlenbeck-compactification). We may furthermore assume that the restriction to any $\tau \in[0,1]$ is transversal.

Let us denote the perturbation parameter by $p \in \mathscr{P}$, and let us further assume that the restriction of the parametrised moduli space to $(\tau, p)$, denoted by $M_{\mathfrak{s}, F}(\tau, p)$, is already compact in the main stratum for all $\tau \in[0,1]$ and all 'small enough p'. We shall furthermore assume that $M_{\mathfrak{s}, F}(\tau, p)=M_{\mathfrak{s}, F}^{* *}(\tau, p)$, that is, that no reducibles occur for generic parameters $p$ - this being an admittedly speculative assumption that we don't expect to hold in general, even if $b_{2}^{+}(X)>0$. Then for generic parameter $p$ the moduli space $M_{\mathfrak{s}, F}(\tau, p)$ is a smooth closed manifold of the expected dimension for generic perturbation $p$.

By the usual argument the closed manifolds $M_{\mathfrak{s}, F}(\tau, p)$ and $M_{\mathfrak{s}, F}(0, p)$ are then cobordant. However, it is a rather easy observation that the moduli spaces $M_{\mathfrak{s}, F}(0, p)$ are generically empty. In fact, for parameter $\tau=0$ the map $\mu_{0, \tau}$ is traceless, so the trace of the curvature equation of (8) for $(A, \Psi)$ becomes:

$$
F_{\operatorname{det}(A)}^{+}=p r_{\Lambda_{+}^{2} \otimes i \mathbb{R}}(p) .
$$

This is a perturbed abelian $A S D$ equation. As the derivative of the 'map' $F^{+}$on $U(1)$ connections is given by $d^{+}: \Omega^{1}(X ; i \mathbb{R}) \rightarrow \Omega_{+}^{2}(X ; i \mathbb{R})$, and as the cokernel of this map has dimension $b_{2}^{+}(X)$ we see that for $b_{2}^{+}(X)>0$ the space of solutions $(A, \Psi)$ to (14) is empty for generic parameter $p \in \mathscr{P}$.

\section{4. $U(n)$ MODULI SPACES ON KäHLER SURFACES}

In classical Seiberg-Witten theory Kähler surfaces are of a significant importance. Indeed, they provided the first examples of 4-manifolds with non-trivial SeibergWitten invariants [29. This was generalised to symplectic manifolds [?]. All other non-vanishing results known to the author are derived from these manifolds by various kinds of glueing results for the Seiberg-Witten invariants [?], [?].

As the $U(n)$ monopole equations are a generalisation of the classical SeibergWitten equations it is therefore most natural to study the $U(n)$ monopole moduli spaces for Kähler surfaces. Whereas the analysis of the $U(n)$ monopole equations on Kähler surfaces is very analogous to the classical situation the final conclusion is in sharp contrast to the classical situation. Indeed, we will show in Corollary 4.5 that if we perturb the monopole moduli space on a Kähler surface with a non-vanishing holomorphic 2-form then the associated moduli space is empty.

Non-abelian monopoles on Kähler surfaces have also been studied by Teleman [26], Okonek and Teleman [22] and by Bradlow and Garcia-Prada [2], but with a rather complex geometric motivation. Corollary 4.5 seems to appear here for the first time. 
4.1. The $U(n)$ - monopole equations on Kähler surfaces. We will quickly recall now the canonical $S p i n^{c}$ - structure on an almost complex surface. The additional condition of $X$ being Kähler implies that there is a canonical Spin ${ }^{c}$ connection induced by the Levi-Civita connection. This will be our fixed background Spin $^{c}$ connection and it is then simple to determine the Dirac-operator associated to this fixed connection and a $U(n)$ - connection in a Hermitian bundle $E$. We will then write down the $U(n)$ monopole equations in this particular setting.

Suppose we have an almost complex structure $J: T X \rightarrow T X$ on the closed, oriented Riemannian 4-manifold $X$ which is isometric. The associated Kähler form $\omega$ is defined by the formula

$$
\omega_{g}(v, w):=g(J v, w) .
$$

This is an anti-symmetric form of type $(1,1)$ when extended to the complexification $T X^{\mathbb{C}}:=T X \otimes_{\mathbb{R}} \mathbb{C}$. It is a fundamental fact that the complexification of the bundle of self-dual two forms is given by

$$
\Lambda_{+}^{2} \otimes \mathbb{C}=\mathbb{C} \omega_{g} \oplus \Lambda^{2,0} \oplus \Lambda^{0,2} .
$$

Let $e(u)$ denotes exterior multiplication with the form $u \in \Lambda\left(T^{*} X^{\mathbb{C}}\right)$ and $e^{*}(u)$ its adjoint with respect to the inner product induced by the Riemannian metric.

There is a canonical Spinc-structure associated to an almost-complex structure $J$ on $X[12$. We shall denote it by $\mathfrak{c}$. The spinor bundles are defined to be

$$
\begin{aligned}
& S_{\mathfrak{c}}^{+}:=\Lambda^{0,0}(X) \oplus \Lambda^{0,2}(X), \\
& S_{\mathfrak{c}}^{-}:=\Lambda^{0,1}(X),
\end{aligned}
$$

and the Clifford multiplication is given by

$$
\begin{gathered}
\gamma: \Lambda^{1}\left(T^{*} X\right) \rightarrow \operatorname{Hom}_{\mathbb{C}}\left(S_{\mathfrak{c}}^{+}, S_{\mathfrak{c}}^{-}\right) \\
u \mapsto \sqrt{2}\left(e\left(u^{0,1}\right)-e^{*}\left(u^{0,1}\right)\right) .
\end{gathered}
$$

The induced isomorphism

$$
\gamma: \Lambda_{+}^{2}(X) \otimes \mathbb{C} \rightarrow \mathfrak{s l}\left(S_{\mathfrak{c}}^{+}\right)
$$

is then seen to be given by the formula

$$
\gamma\left(\eta^{1,1}+\eta^{2,0}+\eta^{0,2}\right)=4\left(\begin{array}{cc}
-i \Lambda_{g}\left(\eta^{1,1}\right) & -*\left(\eta^{2,0} \wedge-\right) \\
\eta^{0,2} & i \Lambda_{g}\left(\eta^{1,1}\right)
\end{array}\right) .
$$

Here we use the commonly used convention to denote contraction with $\omega_{g}$, that is $e^{*}\left(\omega_{g}\right)$, by the symbol $\Lambda_{g}$.

Now suppose that $X$ is a Kähler surface. This means that first the almost complex structure $J$ is integrable to a complex structure, and second that the Kähler form $\omega_{g}$ is closed, $d \omega_{g}=0$. The condition of closedness implies (cf. [?], p. 148) that the the almost complex structure $J$ is parallel with respect to the Levi-Civita-connection $\nabla_{g}$. As a consequence, the splittings

$$
\Lambda^{k}(X) \otimes \mathbb{C}=\oplus_{p+q=k} \Lambda^{p, q}(X)
$$

are $\nabla_{g}$-parallel, where we also denote by $\nabla_{g}$ the connection induced by the LeviCivita connection on all exteriour powers of $T^{*} X$. The canonical $\operatorname{Spin}^{c}$-connection is now simply given by the the connection $\nabla_{g}$ in the bundles $\Lambda^{0,0}, \Lambda^{0,1}$ and $\Lambda^{0,2}$. 
Let $E$ be a Hermitian vector bundle on $X$, and further $\nabla_{A}$ a unitary connection on $E$. We shall use the notation convention $\Lambda^{p, q}(E):=\Lambda^{p, q}(X) \otimes E$, and by $\Omega^{p, q}(E)$ we shall denote the space of sections of the latter bundle, $\Omega^{p, q}(E)=\Gamma\left(\Lambda^{p, q}(E)\right)$.

Definition 4.1. The operator $\bar{\partial}_{A}: \Omega^{p, q}(E) \rightarrow \Omega^{p, q+1}(E)$ is defined to be the composition of $d_{A}: \Omega^{p+q}(E) \rightarrow \Omega^{p+q+1}(E)$, the extension of the exteriour derivative to forms with values in $E$ by means of the connection $\nabla_{A}$, with the bundle projection $\Lambda^{p+q+1}(E) \rightarrow \Lambda^{p, q+1}(E)$.

The Dirac operator associated to the canonical $\operatorname{Spin}^{c}$-connection $\nabla_{g}$ in the canonical ppin $^{c}$-structure $\mathfrak{s}_{c}$ and the unitary connection $A$ in the Hermitian bundle $E$ is expressible in terms of the above operator $\bar{\partial}_{A}$ and its formal $L^{2}$-adjoint $\bar{\partial}_{A}^{*}$ as follows:

$$
\not D_{A}^{+}=\sqrt{2}\left(\bar{\partial}_{A}+\bar{\partial}_{A}^{*}\right)
$$

This is a well-known fact in the case $n=1$ [12]. The proof of the general case follows along the same lines. In particular, the proof given in the lecture notes 28$]$ is directly applicable to our situation.

We will now study the $U(n)$ monopoles associated to the data $(\mathfrak{c}, E)$ with spinor bundles $W_{\mathrm{c}, E}^{ \pm}=S^{ \pm} \otimes E$. Note that, up to tensoring $E$ with a line bundle, we can always assume that general data $(\mathfrak{s}, E)$ is of the particular form $(\mathfrak{c}, E)$. Now according to the isomorphism $W_{\mathfrak{c}, E}^{+} \cong \Lambda^{0,0}(E) \oplus \Lambda^{0,2}(E)$ a spinor $\Psi \in \Gamma\left(X ; W_{\mathfrak{c}, E}^{+}\right)$ can be written as $\Psi=(\alpha, \beta)$ with $\alpha \in \Omega^{0,0}(X ; E)$ a section of $E$ and $\beta \in \Omega^{0,2}(X ; E)$ a 2-form of type $(0,2)$ with values in $E$. We introduce the following notations. We denote by ${ }^{-}: \Lambda^{p, q}(E) \rightarrow \Lambda^{q, p}\left(E^{*}\right)$ the conjugate linear isomorphism which is the tensor product of complex conjugation on the forms and the conjugate linear isomorphism specified by the hermitian structure on the bundle $E$. We denote by * $: \Lambda^{p, q}(E) \rightarrow \operatorname{Hom}\left(\Lambda^{p, q}(E), \mathbb{C}\right)$ the conjugate linear isomorphism specified by the Hermitian structure on $\Lambda^{p, q}(E)$. For an endomorphism $f \in \operatorname{End}(E)$ we denote $\{f\}_{\tau}:=(f)_{0}+\frac{\tau}{n} \operatorname{tr}(f) \operatorname{id}_{E}$, where $(f)_{0}$ denotes the trace-free part of $f$. Thus we simply have $\{f\}_{1}=f$. With this said we can write $\mu_{0, \tau}(\Psi)$ according to the above isomorphism as

$$
\mu_{0, \tau}(\Psi)=\left(\begin{array}{cc}
\frac{1}{2}\left(\left\{\alpha \alpha^{*}\right\}_{\tau}-\{* \beta \wedge \bar{\beta}\}_{\tau}\right) & \left\{\alpha \beta^{*}\right\}_{\tau} \\
\left\{\beta \alpha^{*}\right\}_{\tau} & \frac{1}{2}\left(\left\{\beta \beta^{*}\right\}_{\tau}-\left\{\alpha \alpha^{*}\right\}_{\tau}\right)
\end{array}\right) .
$$

It is worth pointing out here that we have $\beta \beta^{*}=* \beta \wedge \bar{\beta}$ which is true because $\Lambda^{0,2}$ is 1-dimensional. In other words, the two diagonal entries only "look" differently.

With the above formulae (15) we can now write down the monopole equations (8) with parameter $\tau$ and as perturbation the imaginary-valued self-dual 2-form $\eta$ for the pair consisting of the spinor $\Psi=(\alpha, \beta) \in \Gamma\left(X ; \Lambda^{0,0}(E) \oplus \Lambda^{0,2}(E)\right)$ and the connection $A$ in $E$ :

$$
\begin{aligned}
\bar{\partial}_{A} \alpha+\bar{\partial}_{A}^{*} \beta & =0 \\
F_{A}^{0,2} & =\frac{1}{4}\left\{\beta \alpha^{*}\right\}_{\tau}+4 \eta^{0,2} \\
-i \Lambda_{g}\left(F_{A}\right) & =\frac{1}{8}\left\{\alpha \alpha^{*}-*(\beta \wedge \bar{\beta})\right\}_{\tau}-i \Lambda_{g}(\eta) .
\end{aligned}
$$


Indeed, the curvature equation of (8) splits into four equations according to the above splitting, but the two equations resulting from the diagonal entries are equivalent, and, using that $\overline{F_{A}^{0,2}}=-F_{A}^{2,0}$ (here again, ${ }^{-}$denotes the complex-conjugation on the forms and the hermitian adjoint on $\operatorname{End}(E)$ ), the two off-diagonal equations also prove to be equivalent.

4.2. Decoupling phenomena, moduli spaces for $b_{2}^{+}(X)>1$ and holomorphic 2-forms. As mentioned before a lot of the analysis of the classical monopole equations on Kähler surfaces carries over to our situation. Before we consider the perturbed monopole equations we shall first draw some intermediate conclusions from the unperturbed monopole equations. In particular there is a decoupling result completely analogous to the classical situation, interpreting monopoles as 'vortices', c.f. also [2], 26].

Proposition 4.2. Let $X$ be a Kähler surface. Suppose that the configuration $(\Psi, A) \in \Gamma\left(X ; S_{\mathfrak{c}}^{+} \otimes E\right) \times \mathscr{A}(E)$ solves the unperturbed $U(n)$ monopole equations with parameter $\tau \in[0,1]$. If we write the spinor as $\Psi=(\alpha, \beta)$ according to the decomposition $S_{\mathfrak{c}}^{+} \otimes E \cong \Lambda^{0,0}(E) \oplus \Lambda^{0,2}(E)$ then one of the following two statements holds:

(1) The second factor of the spinor vanishes identically, $\beta \equiv 0$. Furthermore the pair $(\alpha, A)$ satisfies the following 'Vortex-type' equations

$$
\begin{aligned}
\bar{\partial}_{A} \alpha & =0 \\
F_{A}^{0,2} & =0 \\
i \Lambda_{g}\left(F_{A}\right) & =-\frac{1}{8}\left\{\alpha \alpha^{*}\right\}_{\tau} .
\end{aligned}
$$

(2) The first factor of the spinor vanishes identically, $\alpha \equiv 0$. Furthermore the pair $(\beta, A)$ satisfies the following equations

$$
\begin{aligned}
\bar{\partial}_{A}^{*} \beta & =0 \\
F_{A}^{0,2} & =0 \\
i \Lambda_{g}\left(F_{A}\right) & =+\frac{1}{8}\left\{\beta \beta^{*}\right\}_{\tau} .
\end{aligned}
$$

Proof: Using the first two of the monopole equations (18) we get:

$$
\bar{\partial}_{A} \bar{\partial}_{A}^{*} \beta=-\bar{\partial}_{A} \bar{\partial}_{A} \alpha=-F_{A}^{0,2} \alpha=-\frac{1}{4}\left\{\beta \alpha^{*}\right\}_{\tau} \alpha
$$

We take the inner product with $\beta$ to get now:

$$
\begin{aligned}
\left(\beta, \bar{\partial}_{A} \bar{\partial}_{A}^{*} \beta\right) & =-\frac{1}{4}\left(\beta,\left\{\beta \alpha^{*}\right\}_{\tau} \alpha\right) \\
& =-\frac{1}{4}\left(|\beta|^{2}|\alpha|^{2}-\frac{1-\tau}{n}\left(\beta, \operatorname{tr}\left(\beta \alpha^{*}\right) \alpha\right)\right) \\
& \leq\left(-\frac{1}{4}+\frac{1-\tau}{4 n}\right)|\alpha|^{2}|\beta|^{2} \\
& \leq 0 .
\end{aligned}
$$

Here we have used the Cauchy-Schwarz inequality, noting also that $\left|\operatorname{tr}\left(\beta \alpha^{*}\right)\right| \leq$ $\left|\beta \alpha^{*}\right|=|\beta||\alpha|$. Integrating now the latter inequality over the whole manifold $\bar{X}$ 
yields the following:

$$
0 \leq\left\|\bar{\partial}_{A}^{*} \beta\right\|^{2} \leq\left(-\frac{1}{4}+\frac{1-\tau}{4 n}\right) \int_{X}|\alpha|^{2}|\beta|^{2} \text { vol }_{g} \leq 0
$$

Thus we get $\bar{\partial}_{A}^{*} \beta=0$ and from the Dirac equation also $\bar{\partial}_{A} \alpha=0$. If further we have $\tau>1-n$ then we see from the last inequality that at any point of the manifold $X$ we have $\alpha=0$ or $\beta=0$. But we have $0=\bar{\partial}_{A}^{*} \bar{\partial}_{A} \alpha=\Delta_{\bar{\partial}_{A}} \alpha$, and because $\Delta_{\bar{\partial}_{A}}$ is an elliptic second order operator with scalar symbol it follows from Aronaszajin's theorem [1] that solutions to $\Delta_{\bar{\partial}_{A}} \alpha=0$ satisfy a unique continuation theorem. Similarly we have $0=\bar{\partial}_{A} \bar{\partial}_{A}^{*} \beta=\Delta_{\bar{\partial}_{A}} \beta$, so the same holds for $\beta$. Therefore, if one of $\alpha$ or $\beta$ vanishes on an open subset of $X$, then it vanishes on the whole of $X$. The conclusions now follow from (18).

Remark. If $\tau \neq 0$ the moduli space $M_{\mathfrak{c}, E}(\tau, 0)$ can only contain either solution with $\alpha \neq 0$ or with $\beta \neq 0$. This follows from taking the trace of the third equation of (18) and then integrating it over the whole manifold. The left hand term yields then the topological quantity $-2 \pi\left\langle c_{1}(E) \smile\left[\omega_{g}\right],[X]\right\rangle$.

On a Kähler surface we have $\Delta=2 \Delta_{\bar{\partial}}$, just reflecting again the compatibility between the complex structure and the Riemannian metric. Therefore the harmonic differential forms are also $\bar{\partial}$-harmonic and vice versa. In particular, we get the following decomposition from the Hodge-theorem:

$$
H_{d R}^{2}(X ; \mathbb{C})=H_{\bar{\partial}}^{2,0}(X) \oplus H_{\bar{\partial}}^{1,1}(X) \oplus H_{\bar{\partial}}^{0,2}(X) .
$$

Corollary 4.3. If there are solutions to the unperturbed $U(n)$-monopole equations associated to the data $(\mathfrak{c}, E)$ and to the parameter $\tau \in[0,1]$, then the image $c_{1}^{\mathbb{R}}(E)$ in real (complex) cohomology of the first Chern-class $c_{1}(E) \in H^{2}(X ; \mathbb{Z})$ is of type $(1,1)$ according to the above decomposition (22).

Proof: Under these conditions there is a connection $A$ on $E$ with $F_{A}^{0,2}=0=F_{A}^{2,0}$. From the Chern-Weil formula we have that $\frac{-1}{2 \pi i}\left[\operatorname{tr}\left(F_{A}\right)\right]=c_{1}^{\mathbb{R}}(E)$. There is a 1-form $\lambda$ such that $\omega:=\operatorname{tr}\left(F_{A}\right)-\bar{\partial} \lambda$ is $\bar{\partial}$-harmonic and this class also represents $c_{1}^{\mathbb{R}}(E)$. We have $\omega^{2,0}=0$ and $\omega^{0,2}=\bar{\partial} \lambda$. But a class is $\bar{\partial}$ - harmonic if and only each component according to $\Omega^{p+q}(X)=\oplus \Omega^{p, q}(X)$ is $\bar{\partial}$ - harmonic. But then the harmonic form $\omega^{2,0}=\bar{\partial} \lambda$ must be zero, as it is a $\bar{\partial}$ - exact form also.

In the classical theory a common perturbation of the monopole equations was to perturb with imaginary-valued self-dual 2-forms $\eta$ such that $\eta^{2,0}$ is a holomorphic form [29] [3. There are such forms with $\eta^{2,0} \neq 0$ precisely if $b_{2}^{+}(X)>1$. We will now consider this type of perturbation in the general case of $U(n)$ monopoles even though these perturbations are not enough to get generic regularity of the moduli space in the case $n>1$. However, it will turn out that the moduli spaces perturbed in this way are empty in the case $n>1$ as soon as the perturbing form $\eta$ is non-zero.

If the unperturbed $U(n)$ monopole moduli space is empty then any invariant derived by the scheme 'evaluation of cohomology classes on the fundamental cycle of the moduli space' should be zero. Indeed, that kind of invariant would be defined with a 'generic' moduli space, i.e. one which is cut out transversally by the suitably 
perturbed monopole equations. An empty moduli space is always generic. Thus if there is a non-trivial invariant derived from some generic moduli space then the associated unperturbed moduli space may be not generic, but it could not be empty. Therefore it is natural to consider topological data $(\mathfrak{s}, E)$ only for situations where the unperturbed $U(n)$ monopole moduli spaces are a priori non-empty. As we have seen, this can only be the case if the first Chern-class $c_{1}^{\mathbb{R}}(E)$ is of type $(1,1)$ according to the decomposition (22). Therefore we shall include this hypothesis to the next two results, the following theorem and its corollary:

Theorem 4.4. Let $X$ be a Kähler surface and let $E$ be a bundle such that its first Chern-class $c_{1}^{\mathbb{R}}(E)$ is of type $(1,1)$. Further let $\eta$ be an imaginary-valued 2form with $\eta^{2,0}$ holomorphic. Then the $U(n)$ - monopole equations (18) associated to the data $(\mathfrak{c}, E)$, to the perturbation form $\eta$, and to the parameter $\tau \in(0,1]$ are equivalent to the following system of equations:

$$
\begin{aligned}
\bar{\partial}_{A} \alpha & =0 \\
\bar{\partial}_{A}^{*} \beta & =0 \\
F_{A}^{0,2} & =0 \\
\frac{1}{4}\left\{\beta \alpha^{*}\right\}_{\tau} & =\eta^{0,2} \\
-i \Lambda_{g}\left(F_{A}\right) & =\frac{1}{8}\left\{\alpha \alpha^{*}-\beta \beta^{*}\right\}_{\tau}-i \Lambda_{g}(\eta)
\end{aligned}
$$

Proof: We will derive the following formula for a solution $((\alpha, \beta), A))$ to the $U(n)$-monopole equations (18) with parameter $\tau$ and perturbation $\eta$ :

$$
\begin{aligned}
0= & 4\left\|F_{A}^{0,2}\right\|_{L^{2}(X)}^{2}+4 \frac{1-\tau}{\tau n}\left\|\operatorname{tr} F_{A}^{0,2}\right\|_{L^{2}(X)}^{2}+\left\|\bar{\partial}_{A}^{*} \beta\right\|_{L^{2}(X)}^{2} \\
& -\frac{4}{\tau}\left\langle 2 \pi i\left[\eta^{2,0}\right] \smile c_{1}(E),[X]\right\rangle .
\end{aligned}
$$

The conclusion then clearly follows as the topological term vanishes by assumption.

Provided that we have $\tau \neq 0$ the endomorphism $\beta \alpha^{*}$ can be expressed as

$$
\begin{aligned}
\beta \alpha^{*} & =\left\{\beta \alpha^{*}\right\}_{\tau}+\frac{1-\tau}{n} \operatorname{tr}\left(\beta \alpha^{*}\right) \\
& =\left\{\beta \alpha^{*}\right\}_{\tau}+\frac{1-\tau}{\tau n} \operatorname{tr}\left(\left\{\beta \alpha^{*}\right\}_{\tau}\right) \\
& =4 F_{A}^{0,2}-4 \eta^{0,2}+4 \frac{1-\tau}{n \tau} \operatorname{tr}\left(F_{A}^{0,2}\right)-4 \frac{1-\tau}{\tau} \eta^{0,2}
\end{aligned}
$$

where the last equation used the second of the monopole equations (18) and the trace of it.

Again we get from the Dirac-equation that $\bar{\partial}_{A} \bar{\partial}_{A}^{*} \beta+F_{A}^{0,2} \alpha=0$, so that after taking the pointwise inner-product with $\beta$ and using the above equation (25) we 
get:

$$
\begin{aligned}
0= & \left(\beta, F_{A}^{0,2} \alpha\right)+\left(\beta, \bar{\partial}_{A} \bar{\partial}_{A}^{*} \beta\right) \\
= & \left(\beta \alpha^{*}, F_{A}^{0,2}\right)+\left(\beta, \bar{\partial}_{A} \bar{\partial}_{A}^{*} \beta\right) \\
= & 4\left|F_{A}^{0,2}\right|^{2}-4\left(\eta^{0,2}, F_{A}^{0,2}\right) \\
& +4 \frac{1-\tau}{n \tau}\left|\operatorname{tr}\left(F_{A}^{0,2}\right)\right|^{2}-4 \frac{1-\tau}{\tau}\left(\eta^{0,2}, F_{A}^{0,2}\right)+\left(\beta, \bar{\partial}_{A} \bar{\partial}_{A}^{*} \beta\right) \\
= & 4\left|F_{A}^{0,2}\right|^{2}+4 \frac{1-\tau}{n \tau}\left|\operatorname{tr}\left(F_{A}^{0,2}\right)\right|^{2}-\frac{4}{\tau}\left(\eta^{0,2}, F_{A}^{0,2}\right)+\left(\beta, \bar{\partial}_{A} \bar{\partial}_{A}^{*} \beta\right)
\end{aligned}
$$

As the next step we will integrate this whole equation over $X$. Beforehand we shall remark that $\eta^{2,0}$ is closed, and therefore the following integral is of topological nature:

$$
\begin{aligned}
\int_{X}\left(\eta^{0,2}, F_{A}^{0,2}\right) \operatorname{vol}_{g} & =\int_{X} \overline{\eta^{0,2}} \wedge * \operatorname{tr}\left(F_{A}^{0,2}\right) \\
& =-\int_{X} \eta^{2,0} \wedge \operatorname{tr}\left(F_{A}^{0,2}\right) \\
& =2 \pi i\left\langle\left[\eta^{2,0}\right] \smile c_{1}(E),[X]\right\rangle
\end{aligned}
$$

With this said the integral of the formula (26) clearly yields the above formula (24).

Corollary 4.5. Let $X$ be a Kähler surface with $b_{2}^{+}(X)>1$ and let $E$ be a bundle such that its first Chern-class $c_{1}^{\mathbb{R}}(E)$ is of type $(1,1)$. Then for any self-dual imaginary valued 2-form $\eta$ with $\eta^{2,0}$ holomorphic and non-zero and constant $\tau \in(0,1]$ the moduli space $M_{\mathfrak{c}, E}(\eta, \tau)$ is empty.

Proof: Under the given hypothesis the preceeding theorem implies that

$$
\left\{\beta \alpha^{*}\right\}_{\tau}=4 \eta^{0,2} \operatorname{id}_{E} \text {. }
$$

But using the definition of $\left\{\beta \alpha^{*}\right\}_{\tau}$ it is a pure matter of linear algebra to check that for $\eta^{0,2} \neq 0$ this is impossible if $n \geq 2$, because the left hand side of the equation (28) can never be a mutliple of the identity, unless $\alpha=0$ or $\beta=0$.

\section{REFERENCES}

[1] N. Aronaszajin, A unique continuation theorem for solutions to elliptic partial differential equations or inequalities of the second order, Journal de Mathématiques Pures et Appliquées (9), 36, (1957), 235-249.

[2] S. Bradlow, O. Garcia-Prada, Non-abelian monopoles and vortices, Lecture Notes in Pure and Appl. Math. 184 (1997), 567-589.

[3] O. Biquard, Les équations de Seiberg-Witten sur une surface complexe non-kählerienne, Comm. Anal. Geom. 6, No.1 (1998), 173-197.

[4] S. Donaldson, Polynomial invariants for smooth four-manifolds, Topology 2, No. 3, (1990), 257-315.

[5] S. Donaldson, P. Kronheimer, The Geometry of Four-Manifolds, Oxford Mathematical Monographs (1990).

[6] P. Feehan, T. Leness, A general SO(3)-monopole cobordism formula relating Donaldson and Seiberg-Witten invariants, Preprint (2002), arxiv:math.DG/0203047. 
[7] P. Feehan, T. Leness, On Donaldson and Seiberg-Witten invariants, Proc. Sympos. Pure Math. 71 (2001) 237-248.

[8] P. Feehan, T. Leness, SO(3) Monopoles, Level-One Seiberg-Witten Moduli Spaces, and Witten's Conjecture in Low Degrees, Topology and its Applications, to appear.

[9] P. Feehan, T. Leness, PU(2)-monopoles. I: regularity, Uhlenbeck compactness, and transversality, Journal of Differential Geometry, 49 (1998), 265-410.

[10] P. Feehan, T. Leness, Witten's conjecture for four-manifolds of simple type, arxiv:math.DG/0609530 (2006).

[11] O. Garcia-Prada, A direct existence proof for the vortex equations over a compact Riemann surface, Bull. London Math. Soc. 26 (1994), 88-96.

[12] N. Hitchin, Harmonic spinors, Advances in Mathematics 14, (1974) 1-55.

[13] P. Kronheimer, Four-manifold invariants from higher rank bundles, Journal of Differential Geometry, No. 70 (2005), 59-112.

[14] P. Kronheimer, T. Mrowka, The genus of embedded surfaces in the projective plane, Math Research Letters 1, No. 1, (1994) 796-808.

[15] P. Kronheimer, T. Mrowka, Embedded surfaces and the structure of Donaldson's polynomial invariants, Journal of Differential Geometry 41, No.3, (1995), 572-734.

[16] M. Marino, G. Moore, The Donaldson-Witten function for gauge groups of rank larger than one, Commun. Math. Phys. 199 (1998), 25-69.

[17] J. Morgan, The Seiberg-Witten Equations and Applications to the Topology of smooth Four-Manifolds, Math. Notes, Princeton Univ. Press, 1996

[18] J. Milnor, J. Stasheff, Characteristic Classes, Annals of Mathematics Studies, Princeton University Press, (1974).

[19] S. Nicolaescu, Notes on Seiberg-Witten Theory, GSM Vol. 28, American Mathematical Society, (2000).

[20] C. Okonek, A. Teleman, Master Spaces and the Coupling Principle: From Geometric Invariant Theory to Gauge Theory, Commun. Math. Phys. 205 (1999) 437-458.

[21] C. Okonek, A. Teleman, Quaternionic Monopoles, Comm. Math. Phys., Vol. 180, No. 2 (1996), 363-388.

[22] C. Okonek, A. Teleman, The coupled Seiberg-Witten equations, vortices and moduli spaces of stable pairs, Int. J. Math., Vol. 6, No. 6 (1995), 893-910.

[23] C. Okonek, A. Teleman, Recent developments in Seiberg-Witten theory and complex geometry, Several complex variables (Berkeley, 1995-1996), Math. Sci. Res. Inst. Publ. 37, Cambridge University Press, 1999, 391-428.

[24] V. Pidstrigach, A. Tyurin, Localization of Donaldson invariants along the Seiberg-Witten classes, preprint dg-ga/9507004

[25] N. Steenrod, The Topology of fibre bundles, Princeton University Press (1951).

[26] A. Teleman, Non-abelian Seiberg-Witten theory and stable oriented pairs, Int. J. of Math. No.4 (1997) 507-535.

[27] A. Teleman, The moduli space of PU(2)-monopoles, Asian J. of Math.

[28] A. Teleman, Introduction à la Théorie de Jauge, Lecture Notes, available at http://www.cmi.univ-mrs.fr/ ${ }^{\text {teleman }}$

[29] E. Witten, Monopoles and Four-manifolds, Math Research Letters 1 No.1 (1994) 809-822.

[30] R. Wells, Differential Analysis on Complex Manifolds, GTM No. 65, Springer, (1980).

[31] R. Zentner, $P h D$ thesis, available online at http://www.cmi.univ-mrs.fr/ ${ }^{\sim}$ zentner/thesis.pdf

[32] R. Zentner, A vanishing result for a Casson-type instanton invariant, preprint (2009), arXiv:0911.2772

Mathematisches Institut, Westfälische Wilhelms-Universität, Einsteinstr. 62, 48149 Münster, Germany

E-mail address: raphael.zentner@math.uni-muenster.de 